\title{
Micro-Capsules in Shear Flow
}

\author{
R Finken, S Kessler and U Seifert \\ II. Institut für Theoretische Physik, Universität Stuttgart, Pfaffenwaldring 57, 70550 \\ Stuttgart, Germany \\ E-mail: useifert@theo2.physik.uni-stuttgart.de
}

\begin{abstract}
This paper deals with flow-induced shape transitions of elastic capsules. The state of the art concerning both theory and experiments is briefly reviewed starting with dynamically induced small deformation of initially spherical capsules and the formation of wrinkles on polymerized membranes. Initially non-spherical capsules show tumbling and tank-treading motion in shear flow. Theoretical descriptions of the transition between these two types of motion assuming a fixed shape are at variance with the full capsule dynamics obtained numerically. To resolve the discrepancy, we expand the exact equations of motion for small deformations and find that shape changes play a dominant role. We classify the dynamical phase transitions and obtain numerical and analytical results for the phase boundaries as a function of viscosity contrast, shear and elongational flow rate. We conclude with perspectives on timedependent flow, on shear-induced unbinding from surfaces, on the role of thermal fluctuations, and on applying the concepts of stochastic thermodynamics to these systems.
\end{abstract}

PACS numbers: 47.15.G- (Low-Reynolds-number (creeping) flows), 87.16.D(Membranes, bilayers, and vesicles), 83.50.-v (Deformation and flow)

Submitted to: J. Phys.: Condens. Matter

\section{Introduction}

Elastic capsules constitute an important class within soft matter research both from a fundamental and application point of view [1, 2, 3, 4, 5]. In contrast to strongly deformable fluid vesicles [6], the membrane of capsules exhibits finite shear elasticity. This structural feature is achieved either by chemically or physically crosslinking the molecules forming the membrane [7] or by attaching a polymeric network to a fluid membrane as in red blood cells (RBCs) [8]. From the perspective of this paper these biological capsules can be subsumed under this heading of a micro-capsule as well. Promising applications of these objects arise interalia from the pharmaceutical perspective of optimized drug encapsulation delivery and release [9, 10].

In a microfluidic context, one of the most relevant issues is the behaviour of these soft objects in externally applied flow. This problem is challenging from a 
theoretical perspective since the shape of the capsule is not given a priori but determined dynamically from a balance of interfacial forces with fluid stresses. Hence, their deformation will depend on their material properties and the type of external flow.

Before reviewing the state of the art concerning theory and experiment in the next two sections, we set the stage by introducing a few key notions for micro-capsule dynamics. As illustrated in figure 1, we consider the dynamics of a single closed elastic membrane that surrounds an inner fluid with viscosity $\eta_{i}$ and is embedded in an ambient flow with viscosity $\eta_{o}$, thereby defining an important quantity, the viscosity contrast $\eta_{i} / \eta_{o}$. In the absence of the capsule we assume a prescribed external flow, in most cases set to be an infinite linear shear flow. For all experimental setups the Reynolds number is small, i.e. dissipation is dominating and inertial effects are negligible. Consequently, the flow is governed by the linear Stokes equations and determined instantaneously by the boundary conditions, where in all cases a no-slip boundary condition is assumed at the membrane.

For describing the capsule state adequately, we define a set of suitable parameters. Capsules and RBCs consist of a thin membrane with a typical thickness in the nanometer range which encloses a fluid on a larger length scale (typically of the order 1-100 $\mu \mathrm{m}$ ) with fixed volume $V$ defining a length scale $R$ as the radius of a sphere with the same volume

$$
V \equiv \frac{4}{3} \pi R^{3}
$$

For capsules with area elasticity, the area $A$ of the membrane, as well as the excess area $\Delta \geq 0$ defined by

$$
A \equiv(4 \pi+\Delta) R^{2}
$$

can change, where $\Delta=0$ corresponds to a sphere. In contrast, the membrane of RBCs and polymerized vesicles has a vanishingly small area compressibility, i.e. $A$ and $\Delta$ are constants. For these area-incompressible objects, shape changes are only possible for initially non-spherical shapes $\Delta>0$.

In most cases considered in this paper, the capsule is roughly ellipsoidal, see figure 1 for a definition of the geometry. The three main dynamical parameters are (i) the inclination angle $\Psi$ between the direction of the shear flow and the long semi-axis, (ii) the phase angle $\Phi$ measuring the angle between the direction of the shear flow and that of the position of a tracer particle which initially was on the endpoint of the long semi-axis and (iii) the Taylor deformation parameter

$$
D \equiv \frac{a_{1}-a_{2}}{a_{1}+a_{2}}
$$

expressed by the lengths of the two semi-axes in the shear plane. For initially spherical capsules the deformation parameter $D$ grows with increasing shear rate, while $D$ reaches a plateau value for capsules with incompressible membrane area at large shear rate.

Two main modes of motion of capsules in shear flow can be identified as illustrated in figure 2: (i) The capsule shape reaches a stationary state in the laboratory frame. 


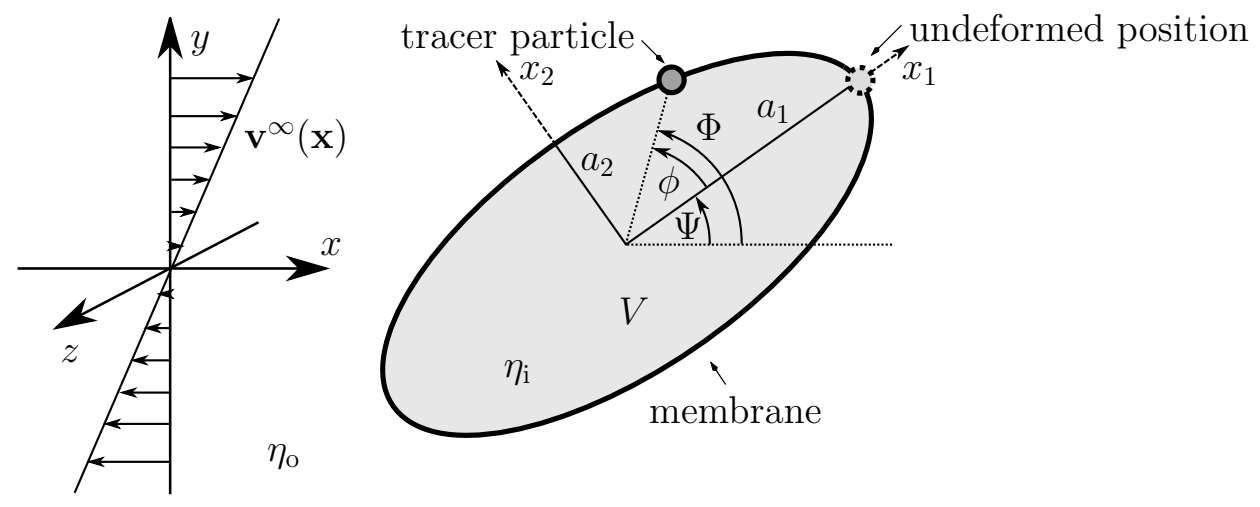

Figure 1. Ellipsoidal capsule with semi-axes $a_{1}, a_{2}, a_{3}$ in linear shear flow $\mathbf{v}^{\infty}$. The shear plane defines the $x y$ plane with shear flow pointing into the $x$ direction. The coordinate axes $x_{1}, x_{2}, x_{3}$ point along the principal directions of the capsule where the $x_{1}$ and $x_{2}$ axes, with $a_{1}>a_{2}$, are chosen to lie in the shear plane and are rotated through the inclination angle $\Psi$ with respect to the $x$ and $y$ axes. The phase angle $\Phi$ measures the angle between the direction of the shear flow and the direction of a tracer particle which initially was on the endpoint of the long semi-axis $a_{1}$. The phase angle $\phi \equiv \Phi-\Psi$ measures the tank-treading motion of the membrane by the angle between the position of the tracer particle with respect to its undeformed position. The membrane encloses a fluid of viscosity $\eta_{i}$ and volume $V$. The fluid outside the capsule has viscosity $\eta_{o}$.

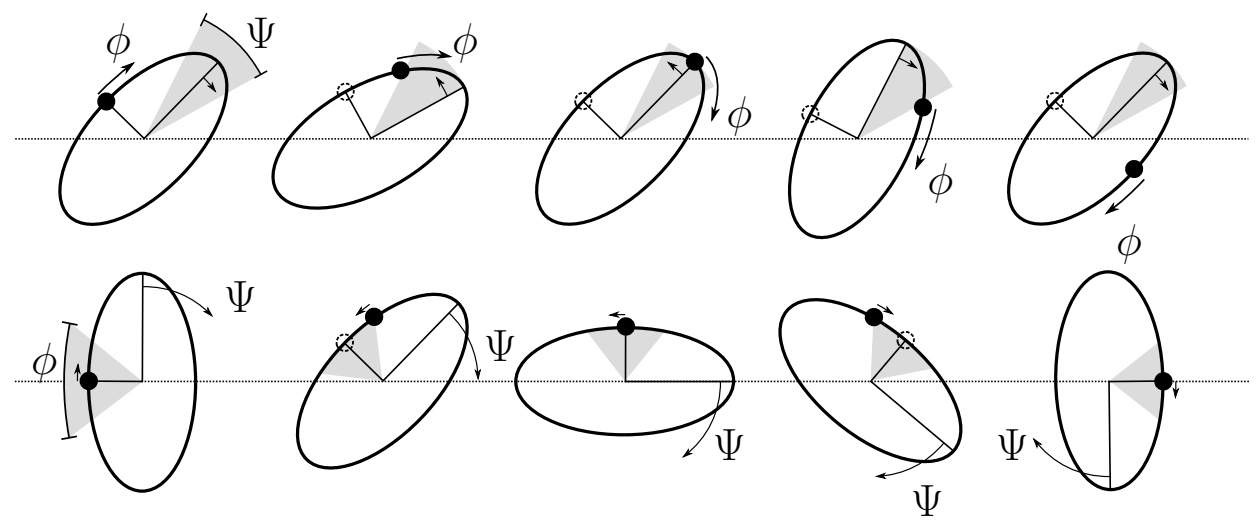

Figure 2. Sequences of swinging (top) and tumbling (bottom) of an ellipsoidal capsule. The black dot and arrow denote the position and motion of a material membrane element. In the swinging motion the inclination angle oscillates around a finite positive inclination angle $\Psi$ while the membrane performs a tank-treading motion with monotonously decreasing phase angle $\phi$. In the tumbling motion the inclination angle $\Psi$ steadily decreases leading to a flipping motion of the capsule and an oscillating phase angle $\phi$. 
The membrane elements rotate along the fixed shape like the chains of a tank. The inclination angle $\Psi$ is then constant, while the phase angle $\phi$ grows monotonously. Such a motion is called tank-treading in its pure form. A variant of this motion with slightly oscillating inclination angle is called swinging and will become important later in this paper. (ii) The capsule shape rotates in the laboratory frame as a rigid body would do. In this case the inclination angle $\Psi$ grows monotonously, while the phase angle $\phi$ stays approximately constant. This motion is called tumbling.

This paper is organized as follows. We give an overview over the theoretical methods in section 2. We then summarize the experimental developments in section 3. The formation of short-wavelength wrinkles on the surface of polymerized capsules is discussed in section 4. We then focus on the dynamics of non-spherical capsules in section 5. where we find the predictions of a simple analytical theory at odds with numerical evidence. To solve this discrepancy in the main original contribution of this paper, we systematically expand the equations of motion in the limit of small capsule deformations in section 6. We find that in certain dynamic regimes shape changes, which are not covered by the simple theory, play an important role in the capsule dynamics. We finish by discussing a few perspectives in section 7. Details of the analytical methods and longer derivations are deferred to appendices $\mathrm{A}-\mathrm{C}$.

\section{Theoretical approaches}

From a theoretical point of view, the full equations of motion are intractable analytically. However, much insight into the physics can be obtained by either studying certain asymptotic limits, where the dynamics can be simplified, or by restricting the number of degrees of freedom to as few as possible. An example of the first strategy is the quasi-spherical expansion of the equations of motion for initially spherical capsules as shown in the following section 2.1. The Keller-Skalak model [11] (described in detail in section 5.1 follows the second strategy, by artificially fixing the shape of the capsule and

only allowing tank-treading and tumbling degrees of freedom. In its original form this model is only applicable for fluid vesicles although it was intended to describe RBCs. It is missing an important energetic contribution to the dynamics, the so-called shape memory [12]. The origin of this term is discussed in section 2.2. An extension of the Keller-Skalak model including the shape memory effect [13, 14] is described in detail in section 5.2. Beyond these two approaches, numerical methods are inevitable. In section 2.3 . we give an overview of the different numerical approaches that have been developed and successfully used to determine the dynamics of vesicles, capsules and RBCs.

\subsection{Initially spherical capsules}

Often the shape of the capsule deviates only little from a sphere. Similarly, in weak flow, initially spherical capsules only deviate slightly from their equilibrium shape. The highly non-linear equations of motion can then be expanded in terms of the small 
deformation vector field. One can then take further advantage of the high symmetry of a sphere by changing to a spherical harmonic representation. A comprehensive treatment is illustrated in [15], where the displacement field is expanded in vector spherical harmonics, which are defined for completeness in Appendix A since we use them in our approach as well.

In early work, Barthès-Biesel [16] considered the steady state of an initially spherical elastic capsule using a small deformation expansion. The membrane is assumed to be homogeneous, isotropic, area-incompressible, and shows no resistance against bending. To linear order in the deformation only tank-treading motions are possible in the long time limit. Only at higher order a transition to tumbling can occur, when the shear forces have deformed the capsule sufficiently. In the steady state, the capsule is orientated with inclination angle $\Psi=\pi / 4$ while the membrane performs a tank-treading motion. With increasing shear rate, the deformation first increases linearly with quadratic corrections. In [17, the relaxational dynamics of a capsule with arbitrary elastic response was obtained still excluding bending energy. There, results have also been obtained in limit cases, like droplets (showing only surface tension) or red blood cells (having a local area incompressibility). More recent theoretical efforts are reviewed by D. Barthès-Biesel and C. Pozrikidis in Chapter 1 and 2, respectively, of [1]. Bending energy was first included for capsules by Rochal et al [15].

\subsection{Shape memory effect: non-spherical capsules}

For a capsule with a spherical equilibrium shape all membrane points are equivalent from a mechanical point of view. This is no longer true for an initially non-spherical capsule such as an RBC, since the long and short axes are distinguished points on the membrane. For a discussion of the consequences for the energetics of a tank-treading motion let us assume that the capsule membrane is unstressed in the equilibrium state. As with tanktreading motion, we now rotate the membrane by the phase angle $\phi$. Since the local geometry of the capsule of each membrane element is different from that of the starting position, the capsule membrane will be sheared, requiring a positive elastic energy. Consequently, a force aims to restore the unstressed equilibrium state. Only after a rotation by $\phi=\pi$ the initial and final position of each membrane point are equivalent to each other due to the mirror symmetry of the ellipsoidal capsule. The membrane is then again unstressed. Any membrane element therefore energetically prefers its initial position (or one of the equivalent positions by symmetry of the reference shape). This property is called shape memory effect [12, and plays an important role also in the dynamics of red blood cells [18, 19, 14, 13].

A direct consequence of the shape memory effect is that capsules perform tumbling

motions for low shear rates. Here, the hydrodynamic forces are not sufficient to overcome the elastic barrier which is present for a hypothetical tank-treading motion. Only for higher shear rates the elastic barrier can be overcome and tank-treading motion becomes possible. Since the force balance of a capsule is also slightly changed by the shape 
memory effect, one now observes stable oscillations of the inclination angle $\Psi$ around a finite value instead of a stationary tank-treading state. This type of motion has therefore been called swinging motion [14]. This term should not be confused with the swinging mode of fluid vesicles, which is also called vacillating-breathing or trembling [20, 21, 22].

\subsection{Full numerical treatment}

For quantitative results beyond asymptotic limits one has to resort to numerical methods for capsules in shear flow. Boundary integral methods (BIM) have been used as first approaches in handling capsules in flow [23, 24, 25, 26, 27, 28, 29, 30, 31, 32, 33. . As a mixture of global spectral methods [34] and boundary integral methods, the spectral collocation [35] and the spectral boundary methods [36, 37, 38] have been developed which use higher order basis functions on a triangulated surface.

The immersed boundary method [39, 40] can be implemented in any hydrodynamic solver. Here, the force density of the boundary is mapped onto a Cartesian mesh. It has been combined with solvers of the Navier-Stokes equation [41, 42] and with lattice Boltzmann methods [43, 44, 45, 46, 47, 48, 49]. The front tracking technique [50] can handle two-phase flows and was used with a finite volume method to treat twodimensional capsules [51].

The oscillations of the shape and orientation of non-spherical capsules have first been observed by Ramanujan et al [28] who used a boundary spectral method. For high and low deformations this method was plagued by numerical instabilities due to the degradation of the grid. Further improvement of the boundary element method allows the stable simulation of swinging and tumbling of highly flattened capsulses only by numerically smoothing the surface [30. More recent approaches, such as the spectral boundary algorithms [36, 37, 38] use higher order basis functions on the triangulated surface to avoid numerical instabilities. Pure spectral methods [34] employ global basis functions to parameterize the capsule membrane. The fluid dynamics part is also simulated using lattice Boltzmann methods [52, 46, 48, 53]. Recently, front-tracking methods have been employed to study the motion of two-dimensional [51] or threedimensional capsules [42, 49].

Existing solvers for the dynamics of vesicles, which treat the flow at a continuum level, either employ the boundary integral method on a discrete triangulation scheme [54, 53, 55] or a phase field or advected field model [56, 57, 58]. An alternative route is provided by stochastic rotation dynamics (SRD) or multi-particle collison dynamics (MPCD), where the flow is modeled using effective fluid particles interacting with a dynamically triangulated membrane [59, 60, 61, 21, 62].

\section{Experiments}

Early experiments [63, 64, 65, 66] confirmed the quasi-spherical predictions for the deformation parameter $D$ (for not too high shear rates) as well as the decreasing 
behaviour of the inclination angle $\Psi$ for increasing shear rate. Although the inclination angle $\Psi$ deviated from the theoretical prediction $\pi / 4$ even in the limit of vanishing shear rates $\dot{\gamma}=0$ [64, 66], experiments together with the quasi-spherical results were used to infer values for the elastic parameters of the membrane.

In contrast to the theoretical predictions for spherical shapes, capsules in such experiments [64, 66] showed oscillations of both the deformation $D$ and the inclination angle $\Psi$. These oscillations caused by the fact that real capsules deviate from a perfectly spherical shape are a direct consequence of the shape memory effect discussed above in section 2.2 ,

In order to observe tumbling motion for experimentally accessible shear rates, capsules which show a large deviation form the spherical shape have to be used. Red blood cells are an ideal and convenient choice. In early experiments, red blood cells have only be examined either in a tumbling state in the blood plasm or in a swinging state for high shear rates showing a stationary inclination angle [67, 68, 69, 70]. The motion for low shear rates and close to the swinging transition was experimentally examined by Abkarian et al [14. Within the swinging regime the frequency of the oscillation of the inclination angle is exactly twice the frequency corresponding to the tank-treading motion. While a membrane point is advected a full rotation along the membrane, the inclination angle is performing two full swinging motions. This relation highlights the direct connection between the oscillations of the shape and the motion of the membrane. For a decreasing shear rate within the swinging regime, the frequency of the oscillation decreases, while the oscillation amplitute of inclination angle increases. The numerical analysis of the full equations of motion using spectral methods [34] discussed in section 2.3 yields qualitative and quantitative agreement with the experimental results. Interestingly, a hysteresis effect at the transition between tumbling and swinging occurs when alternatingly increasing and decreasing the shear rate [14]. Here, a single tumbling motion seems to mix with multiple swinging motions.

\section{Wrinkling}

Rather than deforming to a slightly ellipsoidal shape, certain polymeric capsules show a wrinkling instability while tank-treading in a linear shear flow [66], see figure 3. The wrinkles are caused by a compression-force acting onto the membrane. Although the membrane moves along the stationary shape while tank-treading, both position and direction of the wrinkels remain constant as they are caused by the compression part of the elongational part of the flow. Thin membranes are unstable with respect to compressive forces. In a flat geometry, compression leads to Euler buckling on the largest possible length scale. To produce small length scale wrinkles on a flat membrane one needs a combination of tensile stress along the wrinkles and a non-local geometric constraint 71.

In the small deformation regime this wrinkling instability was analytically explained

in [72] using a separation of length scales, since the capsule radius is much larger than 


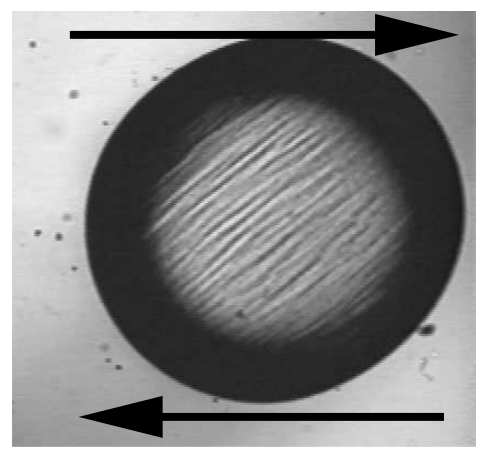

Figure 3. Wrinkling of a polysiloxane capsule $(\simeq 343 \mu \mathrm{m})$ in shear flow as indicated by the arrows (adapted from [66]).
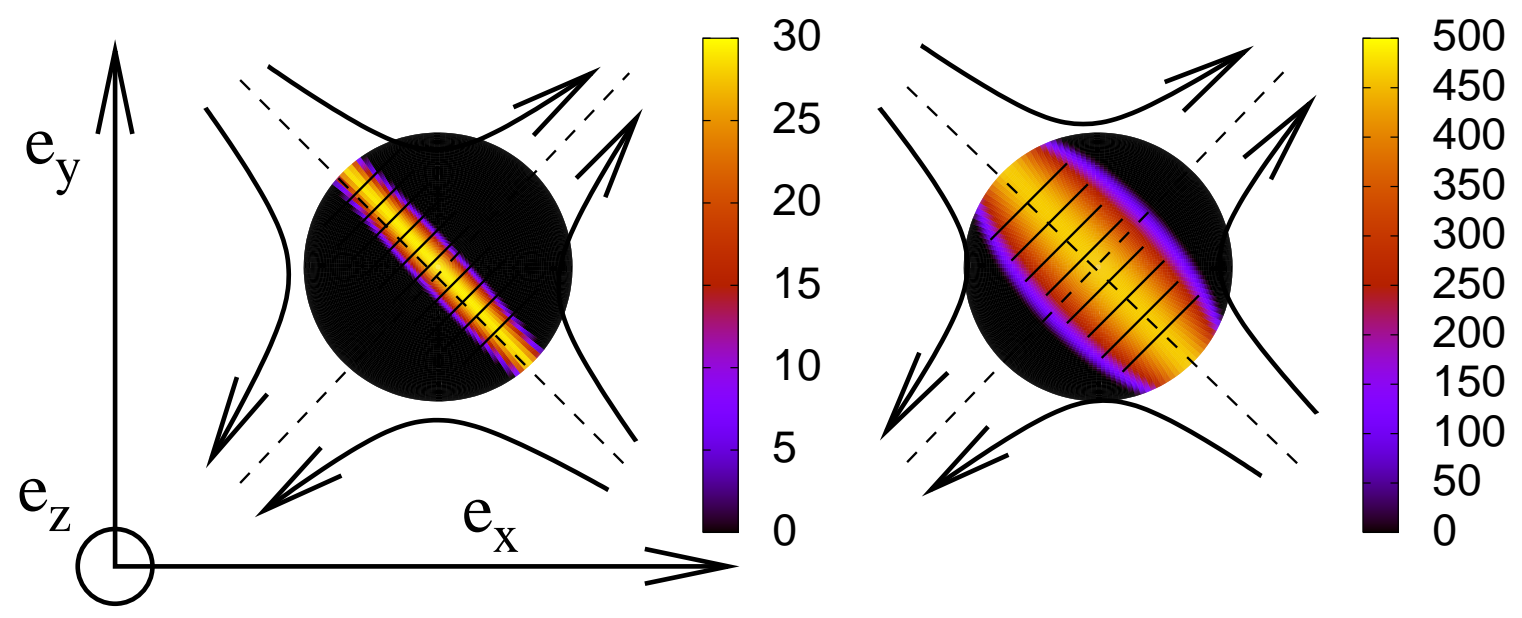

Figure 4. Wrinkling instability region for $\dot{\gamma}=11.5 \mathrm{~s}^{-1}$ (left) and $\dot{\gamma}=17.0 \mathrm{~s}^{-1}$ (right), respectively. The elastic moduli of the membrane are $K=0.2 \mathrm{Nm}^{-1}, \kappa=1 \cdot 10^{-17} \mathrm{Nm}$. In the black coloured region the membrane is still stable with respect to wrinkle formations. Brighter colons indicate a larger growth rate of the wrinkles. Initially, wrinkling occurs only in a small region around the circumference corresponding to the plane of maximal compressive stress. At higher shear rates, this region grows, as does the growth rate of each wrinkling mode. The direction of the wrinkles along the lines of maximal tension are indicated schematically (adapted from [72]).

the typical wavelength of the wrinkles. Even though the overall dynamics of a capsule in viscous flow is inherently non-Hamiltonian, energy considerations dominate the physics at the small length scale of the wrinkles. The energy required to form wrinkles of amplitude $\delta r$ and local wave number $k$ is

$$
\mathcal{H}^{\mathrm{el}} \sim \mathcal{H}_{0}^{\mathrm{el}}+\int d A K(\delta r)^{2} / R^{2}+\sigma_{0}(\delta r)^{2} k^{2}+\kappa(\delta r)^{2} k^{4} .
$$

Here, $K$ is the area incompressibility and $\sigma_{0}<0$ the compression produced by the hydrodynamic force. This term drives the instability. Large wave numbers $k$ are penalized energetically by the bending energy $\sim \kappa k^{4}$. In contrast to a flat membrane, buckling on any length scale on a curved membrane costs elastic energy $\sim K / R^{2}$, since the area of the membrane is increased (decreased) when a membrane element is moved 
outwards (inwards). The resulting tensile (compressive) stress suppresses buckling on too large scales. Only when the compressive force is large enough formation of wrinkles can reduce the elastic energy in the system. This happens at intermediate wave numbers, which can be calculated by minimizing above energy.

For polymerized capsules, the analytical expression for the critical shear rate above which the wrinkling starts reads

$$
\dot{\gamma}_{c}=\frac{8}{5 \eta_{\mathrm{o}} R^{2}} \sqrt{\mu \kappa \frac{K}{K+\mu}},
$$

and the critical wavelength of the wrinkles becomes

$$
k_{c}=\sqrt[4]{\frac{4 \mu}{R^{2} \kappa} \frac{K}{K+\mu}} .
$$

At $\dot{\gamma}_{c}$, the wrinkles start to form along a circumference which forms an angle of $\pi / 4$ with respect to the shear direction. At higher shear rates $\dot{\gamma}>\dot{\gamma}_{c}$, wrinkling extends over larger regions on the capsule, as shown in figure 4. The analytical expression for the critical shear rate would allow for an experimental determination of the bending rigidity of the membrane which is difficult to obtain otherwise. So far, experimental work seems not to have taken up this suggestion.

\section{Reduced model with two degrees of freedom}

We now turn to capsules which are initially non-spherical.

\subsection{Keller-Skalak model}

In the theory of Keller and Skalak [11, a red blood cell (RBC) is treated within a simplified model. Since as an essential ingredient the shape memory effect of RBCs is missing within this simplified description, the model does not capture essential parts of the physics. Yet, for vesicles the Keller-Skalak model delivers quite a good description. Since it is also the basis for the further modelling of capsules, we briefly recall its main

features. The three-dimensional RBC or vesicle is assumed to have a fixed ellipsoidal shape

$$
\left(x_{1} / a_{1}\right)^{2}+\left(x_{2} / a_{2}\right)^{2}+\left(x_{3} / a_{3}\right)^{2}=1,
$$

see figure 1 .

The (Cartesian) components of the undisturbed shear flow are $(\dot{\gamma} y, 0,0)$. In order to mimic a tank-treading motion of the membrane, the velocity field at the membrane is assumed to have components

$$
\mathbf{v}=\omega_{\mathrm{tt}}^{\mathrm{KS}}\left(-\left(a_{1} / a_{2}\right) x_{2},\left(a_{2} / a_{1}\right) x_{1}, 0\right),
$$

where $\omega_{\mathrm{tt}}^{\mathrm{KS}}$ is a parameter having the dimensions of a frequency. The energy supplied by the external flow has to be balanced with the energy dissipated inside the RBC. 
Likewise the torques acting on the RBC have to balance. The equation of motion of the $\mathrm{RBC}$ derived from this torque balance reads [1]

$$
\dot{\Psi}=-\left(\frac{\dot{\gamma}}{2}+\frac{2 a_{1} a_{2}}{a_{1}^{2}+a_{2}^{2}} \dot{\phi}\right)+\frac{a_{1}^{2}-a_{2}^{2}}{a_{1}^{2}+a_{2}^{2}} \frac{\dot{\gamma}}{2} \cos (2 \Psi) .
$$

The dissipated energy must come from the work performed by the external flow, which leads to the equation

$$
\eta_{i} f_{1} \dot{\phi}^{2}=\eta_{o}\left(f_{2} \dot{\phi}^{2}+f_{3} \dot{\gamma} \dot{\phi} \cos (2 \Psi)\right),
$$

where the geometric quantities $f_{i}$ depend only upon the ratios of the axis lengths $a_{i}$ and are defined by

$$
\begin{aligned}
& \alpha_{i} \equiv a_{i}\left(a_{1} a_{2} a_{3}\right)^{-1 / 3} \text { for } i=1,2,3, z_{1} \equiv \frac{1}{2}\left(\frac{a_{1}}{a_{2}}-\frac{a_{2}}{a_{1}}\right), \\
& z_{2} \equiv\left(\alpha_{1}^{2}+\alpha_{2}^{2}\right) \int_{0}^{\infty}\left(\alpha_{1}^{2}+s\right)^{-3 / 2}\left(\alpha_{2}^{2}+s\right)^{-3 / 2}\left(\alpha_{3}^{2}+s\right)^{-1 / 2} \mathrm{~d} s, \\
& f_{1} \equiv 2 z_{1}^{2}, f_{2} \equiv 4 z_{1}^{2}\left(1-\frac{2}{z_{2}}\right), f_{3} \equiv-4 \frac{z_{1}}{z_{2}} .
\end{aligned}
$$

Both balance laws can be simplified to

$$
\begin{aligned}
\dot{\Psi} & =\dot{\gamma}(A+B \cos (2 \Psi)), \\
\dot{\phi} & =\dot{\gamma} C \cos (2 \Psi)
\end{aligned}
$$

with

$$
\begin{aligned}
A & \equiv-1 / 2, \\
B & \equiv \frac{a_{1} a_{2} f_{3}+\left(f_{2}-f_{1} \eta_{i} / \eta_{o}\right)\left(a_{1}^{2}-a_{2}^{2}\right)}{2\left(a_{1}^{2}+a_{2}^{2}\right)\left(f_{2}-f_{1} \eta_{i} / \eta_{o}\right)}, \\
C & \equiv-\frac{f_{3}}{f_{2}-f_{1} \eta_{i} / \eta_{o}} .
\end{aligned}
$$

The phase diagram for the dynamics of RBCs or vesicles is rather simple in the Keller-Skalak model. The transition between tumbling and tank-treading is independent of the shear rate. For given half axes $a_{i}$, there is a critical viscosity contrast $\left(\eta_{i} / \eta_{o}\right)_{\mathrm{c}}$ given by $|B|=|A|=1 / 2$, below which the RBC or vesicle performs a tank-treading motion. For larger viscosity contrast, it undergoes a tumbling motion. The transition from tumbling to tank-treading is thus independent of the shear rate $\dot{\gamma}$ within the Keller-Skalak model.

For low viscosity contrast $\eta_{i} / \eta_{o}<\left(\eta_{i} / \eta_{o}\right)_{\mathrm{c}}$, i.e. $|B|>1 / 2$, one obtains a steady tank-treading angle

$$
\Psi_{\mathrm{tt}}=\frac{1}{2} \arccos \left(\frac{1}{2 B}\right)
$$

and a steady tank-treading frequency

$$
\omega_{\mathrm{tt}}^{\mathrm{KS}}=\dot{\gamma} \frac{C}{2 B}
$$

For high viscosity contrast $\eta_{i} / \eta_{o}>\left(\eta_{i} / \eta_{o}\right)_{\mathrm{c}}$, i.e. $|B|<1 / 2$, one obtains a steady tumbling motion with mean tumbling rate

$$
\langle\dot{\Psi}\rangle \equiv \lim _{T \rightarrow \infty} \frac{1}{T} \int_{0}^{T} \dot{\Psi}\left(t^{\prime}\right) d t^{\prime}=-\frac{\dot{\gamma}}{2} \sqrt{1-4 B^{2}}
$$




\subsection{Skotheim-Secomb model}

The Keller-Skalak model in its original form can only correctly describe the motion of vesicles, as it does not take into account the shape memory effect. Abkarian et al [14] and Skotheim et al [13] extended the Keller-Skalak model to remedy this deficiency.

The membrane with its elastic property serves as an energy storage and induces an additional torque onto the capsule. The elastic energy $E(\phi)$ required for a hypothetical tank-treading motion must be positive and periodic in the phase angle $\phi$ with period $\pi$, as argued in section 2.2. In [14, 13], the energy was modeled as a simple harmonic function

$$
E(\phi)=E_{0} \sin ^{2} \phi
$$

A Keller-Skalak derivation balancing energy and torque leads to two equations of motion for the two degrees of freedom, the inclination angle and the phase angle.

$$
\begin{aligned}
& \dot{\Psi}(t)=\tilde{A}(t)+\tilde{B} \cos (2 \Psi(t)), \\
& \dot{\phi}(t)=\tilde{C}\left(\cos (2 \Psi(t))-\frac{E_{0}}{V \eta_{o} f_{3} \dot{\gamma}} \sin (2 \phi(t))\right)
\end{aligned}
$$

with constants

$$
\begin{aligned}
\tilde{A}(t) & \equiv-\left(\frac{1}{2} \dot{\gamma}+\frac{2 a_{1} a_{2}}{a_{1}^{2}+a_{2}^{2}} \dot{\phi}(t)\right), \\
\tilde{B} & \equiv \frac{a_{1}^{2}-a_{2}^{2}}{2\left(a_{1}^{2}+a_{2}^{2}\right)} \dot{\gamma} \\
\tilde{C} & \equiv-\frac{f_{3}}{f_{2}-f_{1} \eta_{i} / \eta_{o}} \dot{\gamma} .
\end{aligned}
$$

In dimensionless quantities of shear rate $\chi$, viscosity ratio $\lambda$ and eccentricity $\alpha$

$$
\begin{aligned}
\chi & \equiv \frac{V \eta_{o}\left(-f_{3}\right)}{E_{0}} \dot{\gamma} \\
\lambda & \equiv \frac{f_{1}}{-2 f_{3}} \frac{\eta_{i}}{\eta_{o}}+\frac{-f_{2}}{-2 f_{3}}, \\
\alpha & \equiv \arctan \frac{a_{1}^{2}-a_{2}^{2}}{2 a_{1} a_{2}}
\end{aligned}
$$

the equations of motion can be cast in simple form [73]

$$
\begin{aligned}
& \dot{\Psi}(\tau)=-\cos \alpha \dot{\phi}(\tau)-\lambda(1-\sin \alpha \cos 2 \Psi(\tau)), \\
& \dot{\phi}(\tau)=-\left(\chi^{-1}(\tau) \sin 2 \phi(\tau)+\cos 2 \Psi(\tau)\right) .
\end{aligned}
$$

Here, where $\tau$ is a dimensionless time defined differentially as

$$
d \tau \equiv \frac{2 \dot{\gamma}}{\lambda} d t
$$

Solving the equations of motion within the reduced model for arbitrary parameters has been performed numerically [14, 13, 74]. Kessler et al [73] have obtained analytical solutions in the quasi-spherical limit and, using of the method of asymptotic expansion 

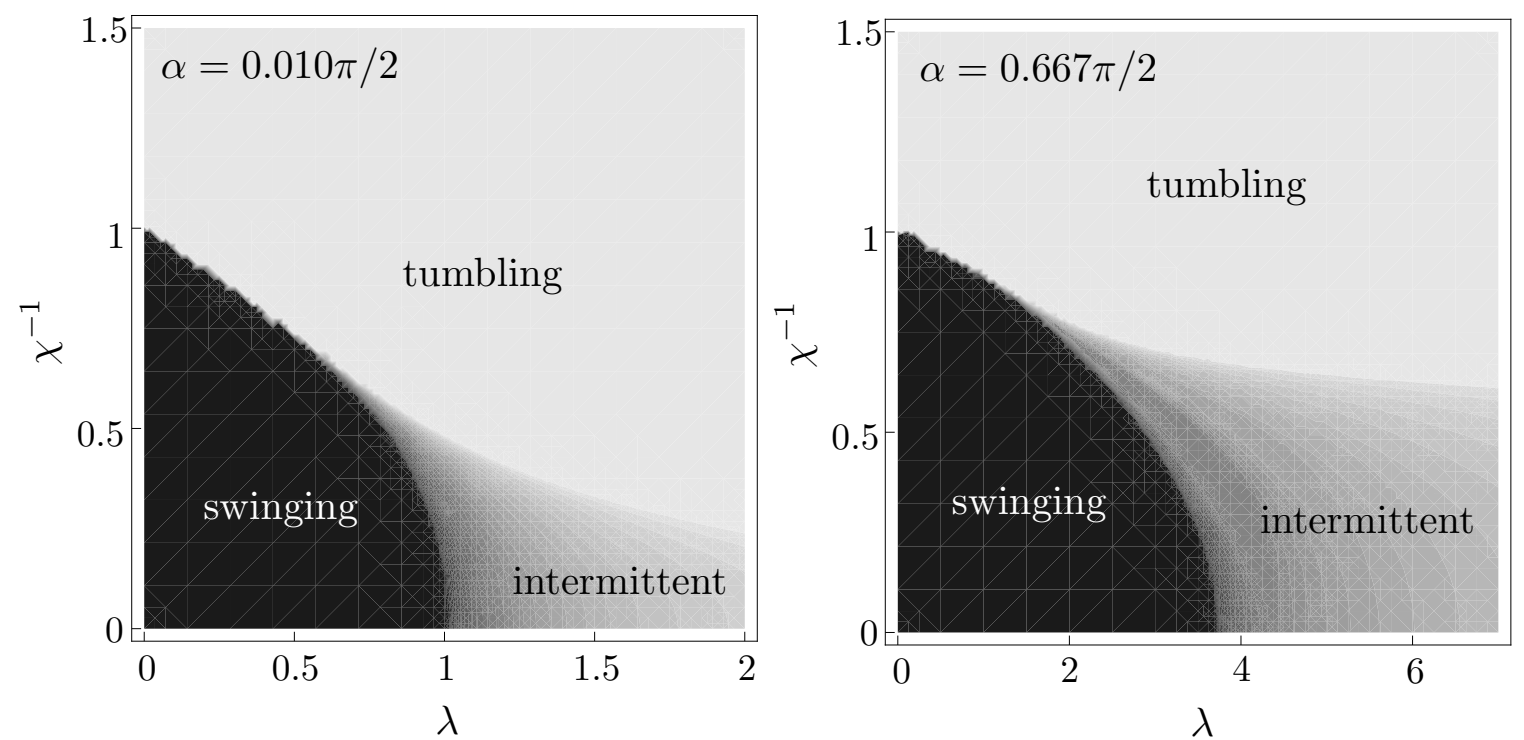

Figure 5. Grey scale plots of the normalized mean tumbling rate $\langle\dot{\Psi}\rangle$ within the reduced model for two different ellipsoidal reference shapes with eccentricity $\alpha$, $\alpha=0$ corresponding to a spherical shape within the shear plane. Dark grey colour corresponds to a vanishing mean tumbling rate $\omega_{\mathrm{tu}}=0$, while light grey corresponds to pure tumbling $\omega_{\mathrm{tu}}=1$.

[75], both the stable trajectories and the slope of the transition between tumbling and swinging.

For small shear rates $\dot{\gamma}$ the capsule always tumbles as the hydrodynamic forces are too weak to overcome the elastic barrier. For increasing shear rate the transition to the intermittent motion takes place. By further increasing the shear rate, the transition to swinging occurs for small and moderate viscosity contrast $\lambda$.

The phase diagram (see figure 5) of this reduced model shows the regimes of the three possible motions. Either the capsule tumbles, swings or performs an intermittent motion with mixed tumbling and swinging periods. The normalized mean tumbling rate

$$
\omega_{\mathrm{tu}} \equiv \frac{\langle\dot{\Psi}\rangle}{\langle\dot{\Psi}\rangle+\langle\dot{\phi}\rangle} \equiv \lim _{T \rightarrow \infty} \frac{\int_{0}^{T} \dot{\Psi}(t) d t / T}{\int_{0}^{T} \dot{\Psi}(t) d t / T+\int_{0}^{T} \dot{\phi}(t) d t / T}
$$

serves as an order parameter to distinguish between the possible motions [73]. Pure tumbling motion is characterized by $\omega_{\mathrm{tu}}=1$, pure swinging by $\omega_{\mathrm{tu}}=0$, while for intermittent motion the mean tumbling rate $\omega_{\text {tu }}$ takes values between 0 and 1 . Noguchi has pointed out that $\omega_{\text {tu }}$ reaches several plateau values between 0 and 1 in the intermittent regime [74.

\subsection{Comparison with spectral method}

To check the validity of the reduced model, its predictions have to be compared with the full non-linear dynamical motion obtained by numerical methods. The first systematic numerical phase diagram for a large range of shear rate and viscosity contrast was constructed using the spectral method described in section 2.3] [34]. Using this method, 


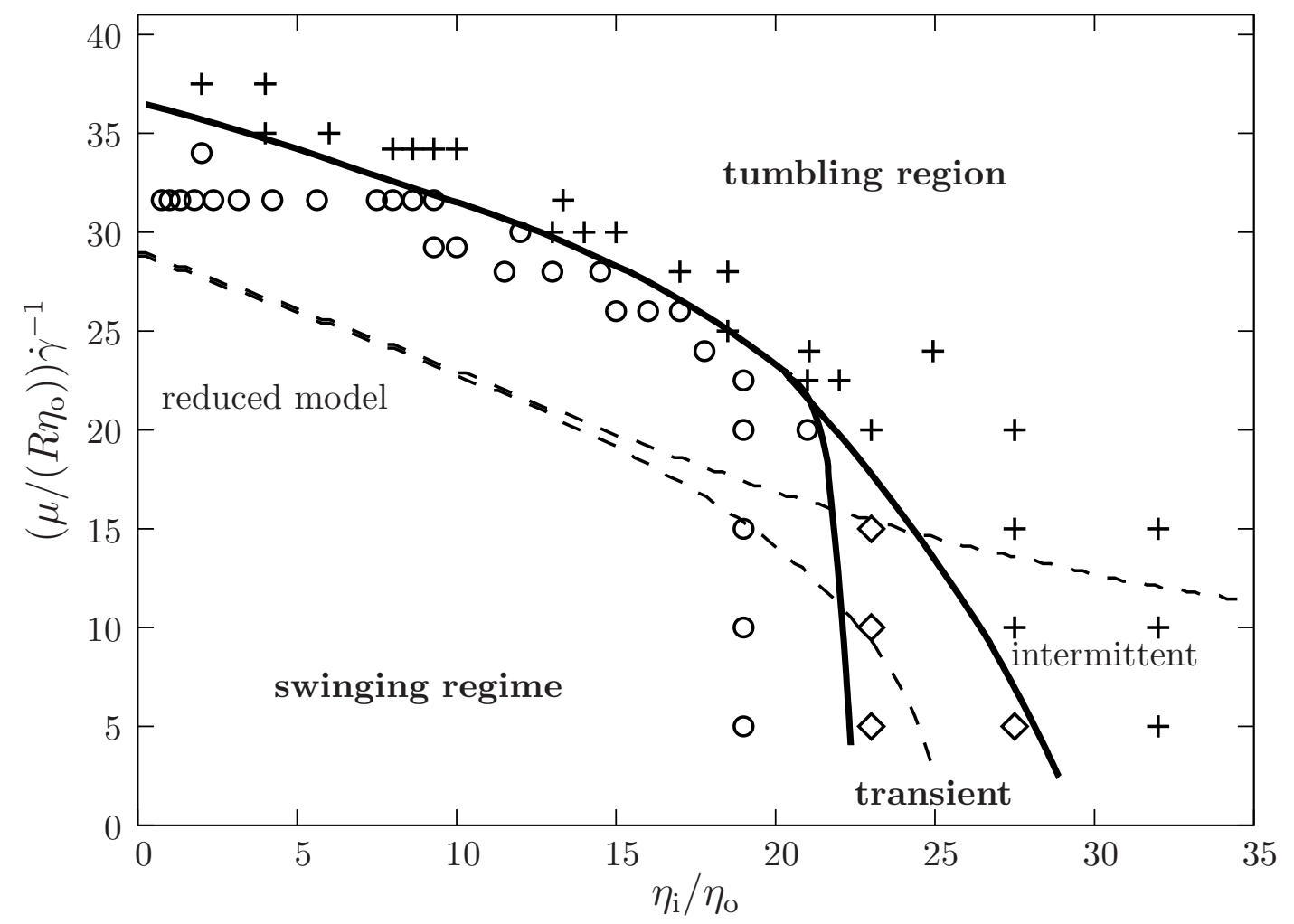

Figure 6. Comparison of phase diagrams deduced by the spectral method 34] (symbols and full line) with the one following from the reduced model (dashed lines). Phase diagrams are functions of the viscosity contrast $\eta_{o} / \eta_{i}$ and the inverse shear rate $\left(R \eta_{o} \dot{\gamma} / \mu\right)^{-1}$, where $\mu$ is the shear modulus of the capsule. For low shear rates the capsule tumbles while for higher shear rates the capsule performs swinging motions for a not too high viscosity contrast. In between these two regimes, there is either a transient regime or intermittent regime deduced by the spectral method or the reduced model, respectively. Adapted from 34.

tumbling motions for capsules slightly deviating from the spherical shape were observed numerically for the first time.

The phase diagram obtained from the spectral method as shown in figure 6 shows qualitative agreement with the predictions of the reduced model. The reduced model correctly predicts a swinging and tumbling motion and a transition for increasing shear rate. In particular for small viscosity ratios, the slopes of the tumbling-swinging transition agree quantitatively, while the absolute position of the transition differs by about 20 percent.

Yet, in the intermittent regime of the reduced model, only transient motion was found in the full model as can be seen in figure 7. Here, the capsule initially tumbles with an steadily decreasing inclination angle $\Psi$, while the phase angle $\phi$ is oscillating. After a few tumbling motions, the capsule starts to perform stable swinging motions, where the inclination angle $\Psi$ oscillates around a stable mean value, while the phase angle $\phi$ is steadily decreasing. Independent studies using two different solvers confirmed this finding: A transient motion relaxing from a tumbling motion into a stable swinging 


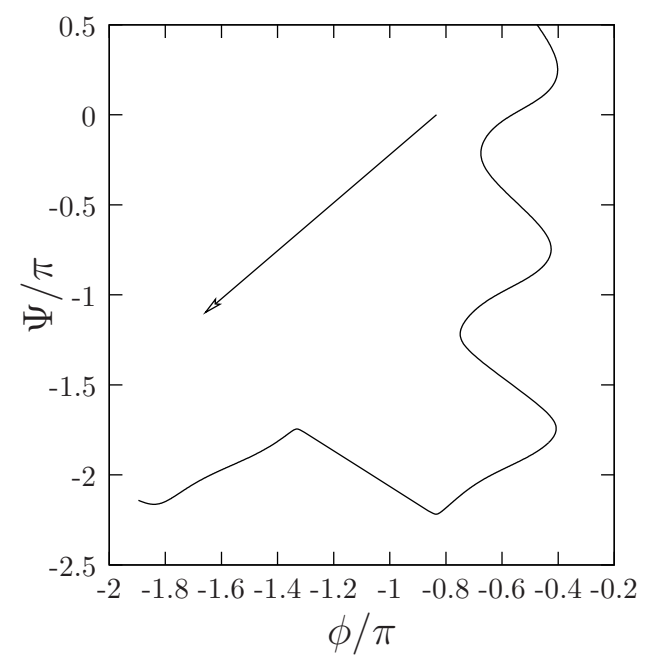

Figure 7. Transient motion from tumbling to stable swinging motion. In this parametric plot the transient trajectory is shown as a function of the inclination angle $\Psi$ and the phase angle $\phi$ where the arrow denotes the direction of time. Initially, the capsule tumbles with oscillating $\phi$ and steadily decreasing $\Psi$, but finally ends up in a swinging motion, where $\Psi$ begins to oscillate around a stable value. Adapted from [34.

motion was always observed in the intermittent regime [47, 42]. We therefore prefer to call the disputed regime transient rather than intermittent 34.

Close to the transition to tumbling, the oscillation of the inclination angle $\Psi$ is very strong, surprisingly leading to temporarily negative values of the inclination angle, as first noted by Kessler et al [34]. Here, the shape changes are very pronounced. This effect was somewhat unfortunately named breathing by Bagchi et al [42]. It should not be confused with the vacillating-breathing motion for vesicles [20] because it does not show pronounced deformations perpendicular to the shear plane. Moreover, the mean inclination angle is not close to zero but finite. Since in all studies shape changes are highly pronounced in the transient regime (see [42] in particular), this reduced model has to be expanded to allow for shape changes to unambiguously clarify the status of the disputed intermittency.

\section{Systematic quasi-spherical expansion}

From the numerical findings it is clear that allowing shape changes is one important ingredient needed to solve the open intermittency question. Noguchi [74] incorporated shape changes within the reduced model heuristically, following extensions of the KellerSkalak model for vesicles [21]. Besides inclination and phase angle, a shape parameter is used as a third degree of freedom. The elastic energy due to shape changes is numerically calculated by stretching a biconcave membrane (with a Skalak energy law). The equation of motion of the shape parameter is deduced by a deformation from the sphere, while the equations of motions for the two angles are obtained by a Keller-Skalak derivation 
[11. The overall phase diagram of the extended model was qualitatively no different from that of the reduced model. In particular, the intermittent motions survived. A temporarily negative inclination angle $\Psi$ is also found in the expanded model. The effect of the bending energy was studied using the same heuristic approach [76].

\subsection{Equations of motion}

We would like to replace this ad-hoc treatment of shape changes by a systematic expansion of the full equations of motion. Specifically, we expand the equations of motion of the capsule for nearly spherical shapes. While the deformation of the capsule shape from a sphere stays small, the displacement of a membrane element during a tank-treading motion is usually large and cannot be treated as a perturbation. The derivation of the elastic energy and the equations of motion is straightforward, but would distract from the main physics. Details are therefore presented in appendix B and appendix C. From the elastic energy, we derive the elastic forces, which must be balanced by hydrodynamic forces. The hydrodynamic forces have contributions from the externally imposed flow and from the induced velocity field, which must be solved dynamically. No-slip boundary conditions at the membrane and at the external walls uniquely determine the dynamics.

Rather than specializing to linear shear flow, we consider general linear external flow

$$
\mathbf{v}^{\infty} \equiv s\left(x \mathbf{e}_{y}+y \mathbf{e}_{x}\right)+\omega\left(x \mathbf{e}_{y}-y \mathbf{e}_{x}\right)
$$

Here, $s$ is a measure of the strength of the elongational flow component and $2 \omega$ is the vorticity. Linear shear flow of the form $\mathbf{v}^{\infty}=\dot{\gamma} y \mathbf{e}_{x}$ corresponds to $s=\dot{\gamma} / 2$ and $\omega=-\dot{\gamma} / 2$. In a microfluidic four-mill device, which was used to study vesicle dynamics quantitatively [77], it is possible to control $\omega$ and $s$ independently.

For motions with the same mirror symmetry with respect to the $x y$-plane as the external flow we derive the equations of motion for the inclination angle $\Psi$, phase angle $\phi$, and a shape parameter $\beta$. The shape parameter $\beta \in[0, \pi / 2]$ is a measure how much of the excess area is stored in the $m=2$ modes. For $\beta=0$ the capsule is a prolate rotationally symmetric ellipsoid with the long axis in the vorticity $(z)$ direction. For $\beta=\pi / 3$ the capsule is again prolate rotationally symmetric, but with the long axis in

the shear (xy) plane. $\beta$ is connected to the Taylor deformation parameter defined in (3) to lowest order via

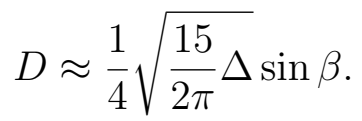

The corresponding shape parameter of the equilibrium shape is denoted with a hat $\hat{\beta}$ (without loss of generality $\hat{\Psi}=\hat{\Phi}=0$ ). The equations of motion as derived in the appendices read

$$
\begin{aligned}
& \dot{\Psi}+\dot{\phi}=-\Lambda \\
& \dot{\phi} \sin \beta=-S^{-1} \sin 2 \phi-\cos 2 \Psi
\end{aligned}
$$




$$
\dot{\beta} \quad=-S^{-1} \cot \hat{\beta} \sin \beta+\cos \beta\left(S^{-1} \cos 2 \phi+\sin 2 \Psi\right),
$$

with

$$
\begin{aligned}
\tau & \equiv 8 \sqrt{\frac{30 \pi}{\Delta}} \frac{s}{32+23 \eta_{i} / \eta_{o}} t, \\
S & \equiv \sqrt{\frac{30 \pi}{\Delta}} \frac{R \eta_{o}}{\mu \sin \hat{\beta}} s, \\
\Lambda & \equiv-\frac{1}{8} \sqrt{\frac{\Delta}{30 \pi}}\left(32+23 \eta_{i} / \eta_{o}\right) \frac{\omega}{s} .
\end{aligned}
$$

Here $\tau$ denotes a dimensionless time and the dot the corresponding derivative. $S$ is a measure of the strength of the elongational flow, and $\Lambda$ measures the relative strength of the rotational flow. In linear shear flow $(s=-\omega=\dot{\gamma} / 2), \Lambda$ depends only on the viscosity contrast and material capsule parameters, while $S$ is proportional to the shear rate $\dot{\gamma}$.

\subsection{Numerical results}

Before we proceed to analyze these equations in more detail, we summarize the findings based on numerically integrating these equations for an initially axisymmetric equilibrium capsule with $\beta(0)=\hat{\beta}=\pi / 3$. In figure 8 , the mean shape parameter $\langle\beta\rangle$ in the long time limit is plotted colour coded as a function of the viscosity contrast $\Lambda$ and the elongational shear rate $S$. In this "dynamical phase diagram", one can distinguish three regions: (i) A swinging region, roughly bounded by $\Lambda<1$ and $S>1$. The mean tumbling rate $\omega_{\mathrm{tu}} \simeq 0$ vanishes in this region, while the shape parameter $\beta \simeq \pi / 2$ is maximal. A typical trajectory over time is shown in figure 9. (ii) A tumbling region roughly bounded by $\Lambda>0.627 S$. Here, $\omega_{\text {tu }} \simeq 1$ takes the highest possible value, while the shape parameter oscillates around the equilibrium one, $\beta \simeq \hat{\beta}$, in the developed tumbling regime. A typical trajectory is shown in figure 10 . (iii) A transient region in between. Here, the shape parameter $\beta \simeq 0$ is small, while the tumbling rate $\omega_{\mathrm{tu}} \simeq 0$ vanishes in the long time limit. A typical trajectory shown in figure 11 reveals that the capsule initially tumbles for a while, before it assumes a stationary swinging state. The initial tumbling time increases with increasing $\Lambda$.

The shape parameter $\beta$ can therefore act as an order parameter for distinguishing the dynamical phases. A cut through the dynamical phase diagram at $S=10$, initially

starting at the equilibrium shape $\beta(t=0)=\hat{\beta}$ is shown in figure 12 . We see that the swinging to tumbling transition at $\Lambda \simeq 1$ is continuous, while $\beta$ jumps at the transient to tumbling transition at $\Lambda \simeq 6.27$, like in a first order phase transition.

\subsection{Analytical results}

After having presented the numerical phenomenology, we analyze the equations of motion analytically and make the connection to the reduced model of section 5, where the shape $\beta$ of the capsule is held fixed. If we ignore the dynamics (37) of the shape 


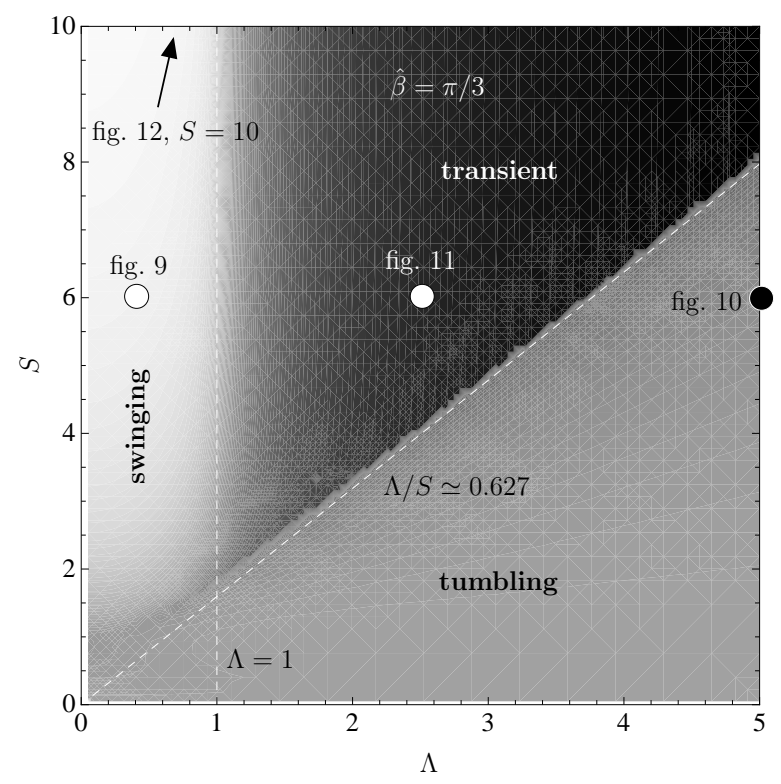

Figure 8. Dynamical phase diagram obtained by numerically integrating 3537 for an initially axisymmetric equilibrium capsule $(\beta(\tau=0)=\hat{\beta}=\pi / 3)$. The mean shape parameter $\langle\beta\rangle$ is shown in a grey scale plot as a function of the shear rate $S$ and the viscosity contrast $\Lambda$. Dark grey corresponds to the minimal value $\langle\beta\rangle=0$, while light grey denotes a maximal value $\langle\beta\rangle=\pi / 2$. Three regimes can be distinguished: swinging, tumbling and a transient motion towards swinging. The small circles correspond to figures 911 , while the cut at $S=10$ through the phase diagram is shown in figure 12. The vertical dashed line corresponds to the analytically obtained phase boundary at $\Lambda=1$ between swinging and transient motion in the limit $S \rightarrow \infty$ with constant $\Lambda$. The inclined dashed white line corresponds to the phase boundary between tumbling and transient motion in the limit $S, \Lambda \rightarrow \infty$ with constant ratio $\Lambda / S \simeq 0.627$.
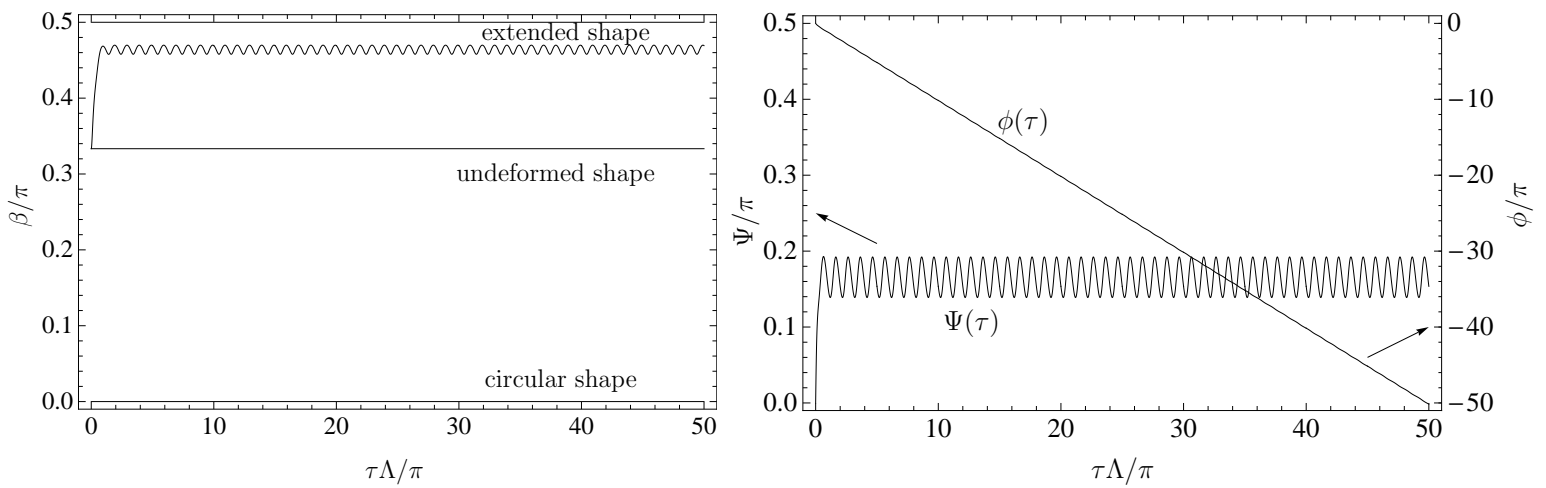

Figure 9. Shape parameter $\beta$ (left), inclination angle $\Psi$, and phase angle $\phi$ (right) as a function of dimensionless time $\tau \Lambda / \pi$ within the swinging regime $(\hat{\beta}=\pi / 3, \Lambda=0.5$ and $S=6.0$, see figure 8 . The shape parameter $\beta$ oscillates around a value $\langle\beta\rangle$ close to $\pi / 2$, implying an almost maximally extended shape within the shear plane. The inclination angle $\Psi$ oscillates around a constant positive value $\langle\Psi\rangle$, while the phase angle $\phi$ linearly decreases with superimposed oscillations (not seen in the plot due to scale). 

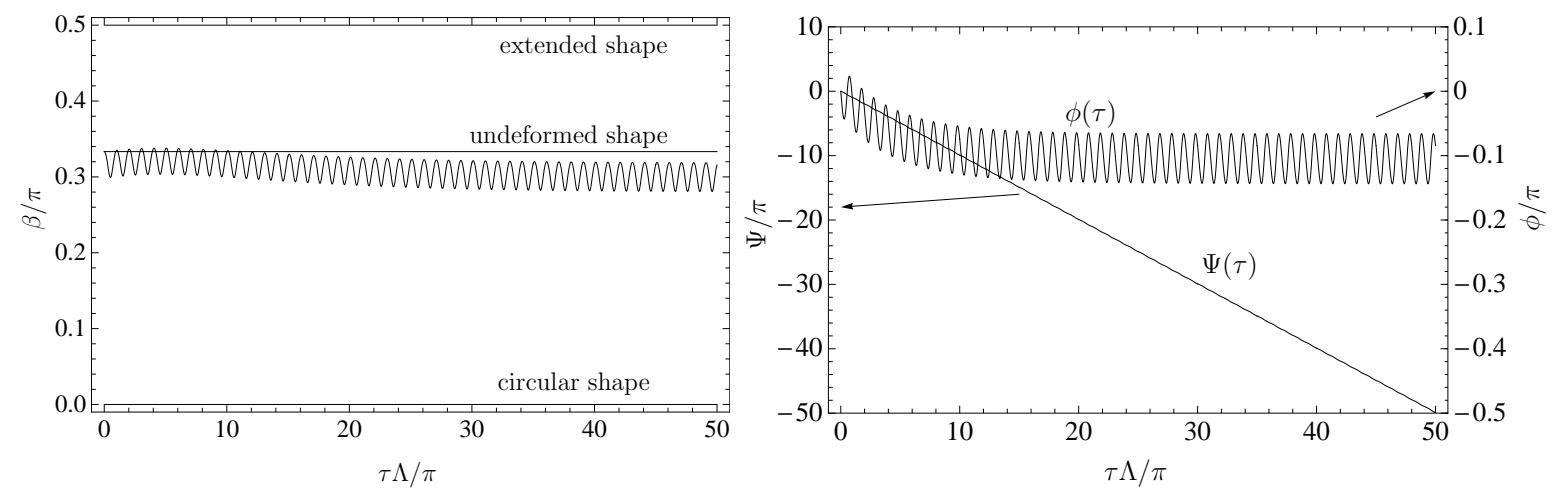

Figure 10. Shape parameter $\beta$ (left), inclination angle $\Psi$, and phase angle $\phi$ and as a function of dimensionless time $\tau \Lambda / \pi$ within the tumbling regime $(\hat{\beta}=\pi / 3, \Lambda=5.0$ and $S=6.0$, see figure 8). The shape parameter $\beta$ oscillates around a value close to its reference value $\hat{\beta}=\pi / 3$. The inclination angle $\Psi$ linearly decreases with superimposed oscillations, while the phase angle $\phi$ oscillates around a constant positive value $\langle\phi\rangle$.
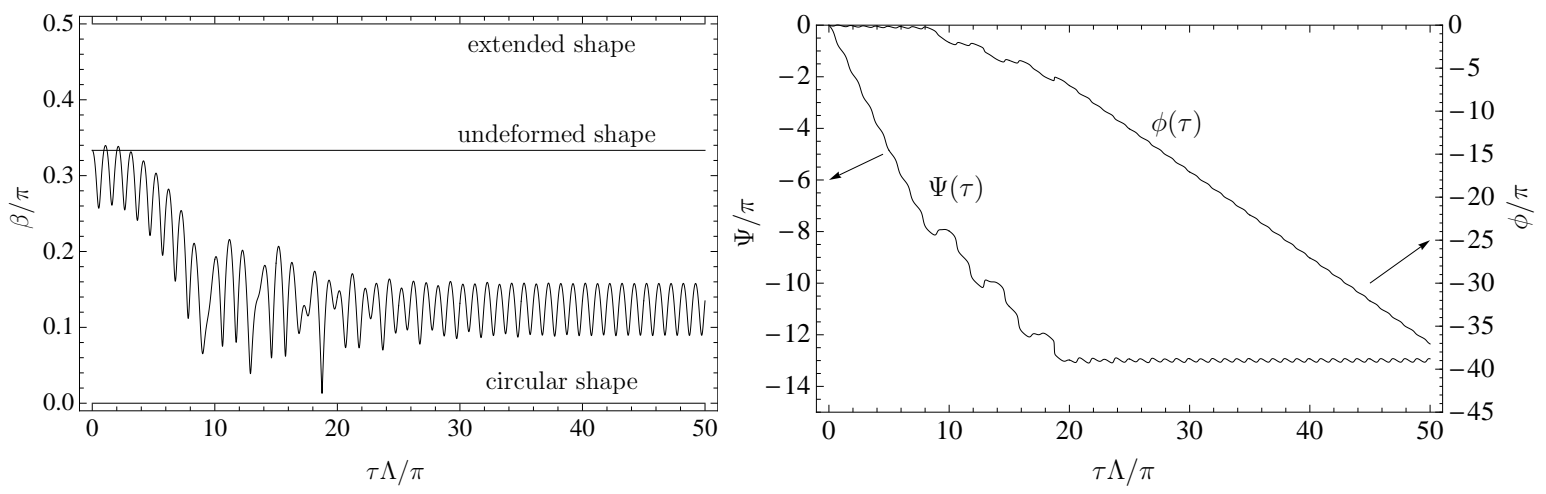

Figure 11. Shape parameter $\beta$ (left), inclination angle $\Psi$, and phase angle $\phi$ (right) as a function of dimensionless time $\tau \Lambda / \pi$ within the transient regime $(\hat{\beta}=\pi / 3, \Lambda=2.5$ and $S=6.0$, see figure 8). Initially, tumbling motions occur where the inclination angle $\Psi$ linearly decreases with superimposed oscillations, while the phase angle $\phi$ oscillates around a constant positive value. On a longer time scale, the shape parameter $\beta$ relaxes to a smaller value, where the capsule is close to circular in the shear plane and strongly extended in the vorticity direction. After the relaxation, the motion changes into a stable swinging, where oscillations are superimposed to a constant mean inclination angle $\langle\Psi\rangle$ and a linear decreasing phase angle $\phi$.

parameter and compare the remaining equations (35, 36) with the corresponding ones in the reduced model, 29,30 , we see that up to a rescaling in time both equations are identical in the quasi-spherical limit $(\alpha \rightarrow 0)$. We identify

$$
\begin{aligned}
\tau_{\mathrm{SK}} & \equiv \tau_{\mathrm{QS}} / \sin (\beta), \\
\lambda & \equiv \Lambda \sin (\beta), \\
\chi & \equiv S .
\end{aligned}
$$

Thus, if the dynamics of the shape parameter $\beta$ is slow compared to that of the angles, we can read off the fast capsule dynamics in the phase diagram of the reduced model, where the effective viscosity ratio $\lambda$ changes slowly in time. We will see that for strong 


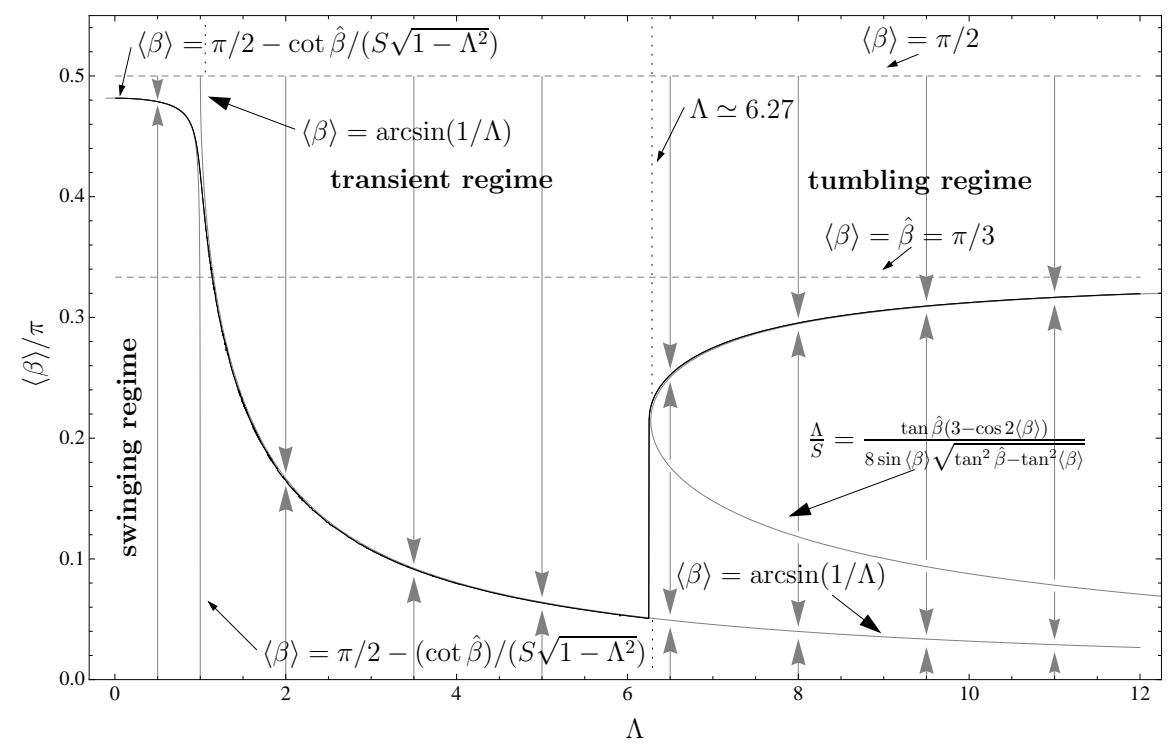

Figure 12. Cut through the phase diagram of figure 8 . The numerically obtained mean shape parameter $\langle\beta\rangle$ is plotted as a function of the viscosity contrast $\Lambda$ at constant shear rate $S=10$ for an axisymmetric capsule starting out of its reference shape $\beta(0)=\hat{\beta}=\pi / 3$ (black line). The grey lines correspond to asymptotic analytical results. For low $\Lambda<1$ the capsule swings with maximally extended shape within the shear plane. For high values $\Lambda>6.27$ the capsule performs tumbling motions with shapes close to the reference shape. In between there is the transient regime where the extension of the shape in the vorticity direction strongly increases with increasing $\Lambda$. The arrows denote the flow towards the long-time stationary value.

flows the shape changes indeed on a much slower time scale than the angles. This separation of time scales is the key to understanding the dynamic phase behaviour of capsules in the quasi-spherical description.

6.3.1. Swinging/transient transition, $S \gg 1, \Lambda$ fixed: We start our analysis by taking the limit of strong flow $S \rightarrow \infty$, while keeping the relative strength of the rotational flow $\Lambda$ constant. The leading order equations then become

$$
\begin{aligned}
\dot{\Psi}+\dot{\phi} & =-\Lambda, \\
(\dot{\Psi}+\Lambda) \sin \beta & =\cos 2 \Psi, \\
\dot{\beta} & =\cos \beta \sin 2 \Psi .
\end{aligned}
$$

We can identify two dynamical regimes.

Swinging: $(\Lambda<1)$ : We now look for stationary tank-treading solutions for the inclination angle $\Psi=\Psi_{0}$ and the shape parameter $\beta=\beta_{0}$. Assuming that $\sin 2 \Psi$ stays positive $\left(0<\Psi_{0}<\pi / 2\right)$, the shape parameter approaches the value $\beta=\pi / 2$ asymptotically. The remaining two equations then reduce to the Keller-Skalak form $\dot{\Psi}=-\Lambda+\cos 2 \Psi$ with the stationary solution for $\Lambda<1$

$$
\Psi_{0}^{\mathrm{sw}}=\frac{1}{2} \arccos \Lambda .
$$


The next order in the inverse shear rate yields oscillating perturbations of the inclination angle

$$
\Psi^{\mathrm{sw}}=\frac{1}{2} \arccos \Lambda+\frac{1}{2 S} \cos 2 \Lambda \tau+\mathcal{O}\left(1 / S^{2}\right),
$$

while the shape parameter is shifted with second order oscillations

$$
\beta^{\mathrm{sw}}=\frac{\pi}{2}-\frac{\cot \hat{\beta}}{S \sqrt{1-\Lambda^{2}}}+\frac{\cot \hat{\beta}}{S^{2} \sqrt{1-\Lambda^{2}}} \sin 2 \Lambda \tau+\mathcal{O}\left(1 / S^{3}\right) .
$$

These results correspond to the numerically observed swinging dynamics. In figure 13 , the numerically calculated long term mean inclination angle $\langle\Psi\rangle$ is compared to the corresponding first term on the right hand side of (48) as a function of $\Lambda$. The amplitude of the oscillatory component, the second term on the right hand side of $(48)$, is compared to numerically obtained data in figure 14 . The amplitude of the second order oscillations of the shape parameter $\beta$ finally is shown in figure 15.

Transient $(\Lambda>1)$ : For $\Lambda>1 \sqrt{44}, 46$ admit a different stationary solution given by

$$
\Psi_{0}^{\mathrm{tr}}=0 \quad \text { and } \quad \beta_{0}^{\mathrm{tr}}=\arcsin (1 / \Lambda) .
$$

These values define the stationary state in the transient regime, which is only reached after initial tumbling motions. The first order calculations add oscillatory terms according to

$$
\begin{aligned}
\phi^{\operatorname{tr}} & =-\Lambda \tau-\frac{\cot \hat{\beta}}{2 S \sqrt{\Lambda^{2}-1}}-\frac{3 \Lambda^{2}-1}{2 S\left(\Lambda^{2}+1\right)} \cos 2 \Lambda \tau+\mathcal{O}\left(1 / S^{2}\right) \\
\Psi^{\mathrm{tr}} & =\frac{\cot \hat{\beta}}{2 S \sqrt{\Lambda^{2}-1}}+\frac{3 \Lambda^{2}-1}{2 S\left(\Lambda^{2}+1\right)} \cos 2 \Lambda \tau+\mathcal{O}\left(1 / S^{2}\right) \\
\beta^{\operatorname{tr}} & =\arcsin 1 / \Lambda+\frac{2 \sqrt{\Lambda^{2}-1}}{S\left(\Lambda^{2}+1\right)} \sin 2 \Lambda \tau+\mathcal{O}\left(1 / S^{2}\right)
\end{aligned}
$$

Again, the mean value of the inclination angle and the oscillation amplitudes of the inclination angle and shape parameter are compared to numerical data in figures $13,15$.

6.3.2. Transient/tumbling transition, $S, \Lambda \gg 1, \Lambda / S$ fixed: In order to discuss the tumbling regime, we must consider a different asymptotic limit. The numerical results indicate that the transient to tumbling transition occurs for large shear rates at fixed ratio $\Lambda / S$. In this limit, two timescales must be considered. On average the parameters vary on a slow timescale $\tau$, but the fast oscillating trigonometric terms produce oscillations on the much faster timescale $\Lambda \tau$. We seek an expansion of $\Psi(\phi, \beta)$ into powers of the small quantity $1 / \Lambda, \Psi \equiv \Psi_{0}+\Psi_{1}+\ldots$. The time derivative of the small oscillating corrections $\Psi_{1}$ are multiplied by $\Lambda$ and can thus contribute to the order one on the left hand side of the equations of motion. The systematic method to analyze processes with different time scales in a perturbation scheme is described in [75]. 


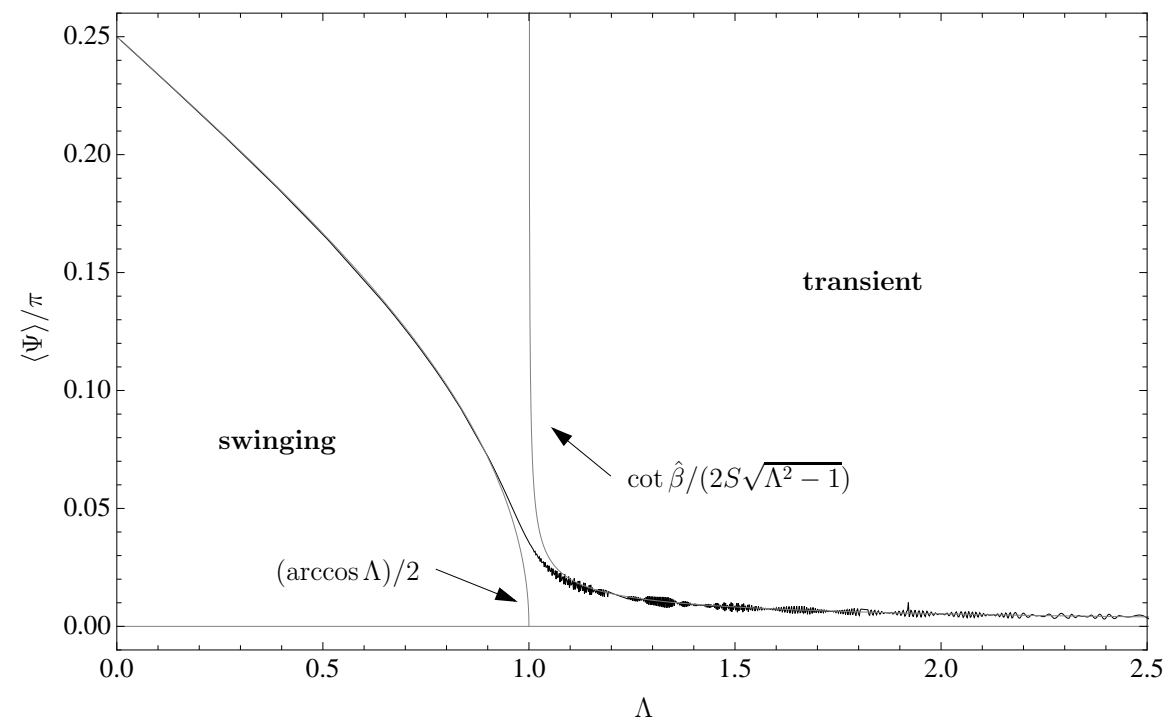

Figure 13. Mean inclination angle $\langle\Psi\rangle$ as a function of the viscosity ratio $\Lambda$ for $\hat{\beta}=\pi / 3$ and $S=10$. The black line corresponds to the numerically obtained values, while the grey lines show analytical results. The mean inclination angle decreases from $\pi / 4$ (corresponding to the direction of the elongational part of the shear flow) at $\Lambda=0$ in the swinging regime. At the transition to the transient regime, the mean inclination angle is close to 0 . In the transient regime, $\langle\Psi\rangle$ asymptotically reaches 0 .

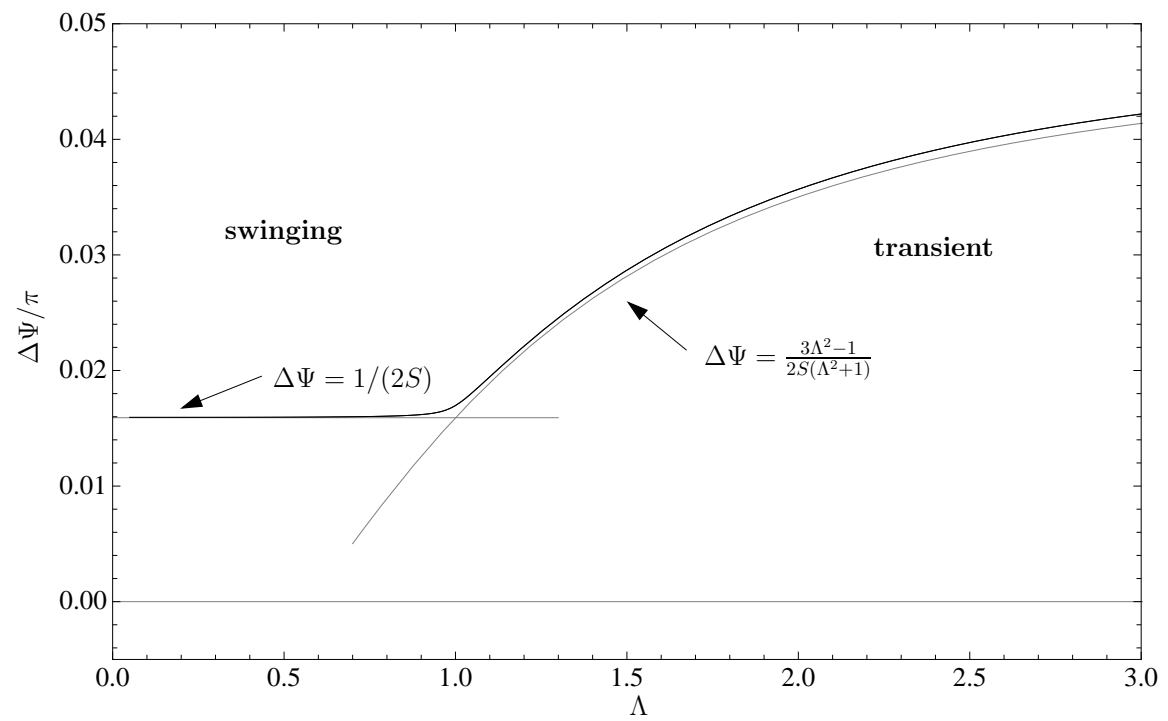

Figure 14. Amplitude $\Delta \Psi$ of the oscillations of the inclination angle $\Psi$ as a function of the viscosity ratio $\Lambda$ for $\hat{\beta}=\pi / 3$ and $S=10$. The black line corresponds to the numerically obtained values, while the grey lines show analytical results.

Long time swinging behaviour: First, we seek to rediscover the stationary tanktreading solution in the transient regime. We expect $\dot{\phi} \simeq-\Lambda$ to leading order. Since only terms of order one occur on the right hand side of $36, \sin \beta$ must be small of order $1 / \Lambda$ on the left hand side. Taking this into account, we recover the tank-treading 


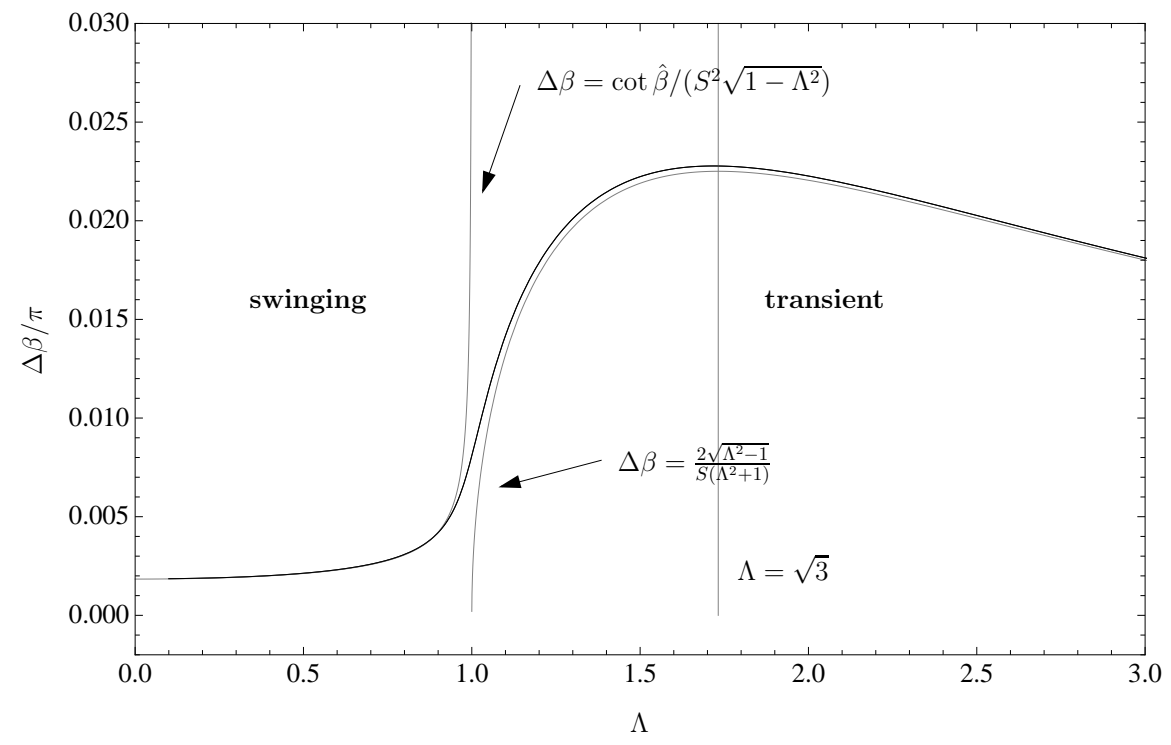

Figure 15. Amplitude $\Delta \beta$ of the oscillations of the shape parameter $\beta$ as a function of the viscosity ratio $\Lambda$ for $\hat{\beta}=\pi / 3$ and $S=10$. The black line corresponds to the numerically obtained values, while the grey lines show analytical results. In the swinging regime, the oscillations show up only in second order in $1 / S$, while in the transient regime, the amplitudes are of order $1 / S$. Shape changes of the swinging capulse are small in the swinging regime and pronounced in the transient regime.

solution $(\arcsin (1 / \Lambda) \approx 1 / \Lambda)$

$$
\Psi_{0}^{\mathrm{sw}}=0 \quad \text { and } \quad \beta_{0}^{\mathrm{sw}}=1 / \Lambda .
$$

Expanding the equations of motion 3535 to the next orders in $1 / S$ gives the oscillatory corrections

$$
\begin{aligned}
\Psi^{\mathrm{sw}} & =\frac{3}{2 S} \cos 2 \Lambda \tau+\mathcal{O}\left(1 / S^{2}\right), \\
\phi^{\mathrm{sw}} & =-\Lambda \tau-\frac{3}{2 S} \cos 2 \Lambda \tau+\mathcal{O}\left(1 / S^{2}\right), \\
\beta^{\mathrm{sw}} & =1 / \Lambda+\frac{2}{\Lambda S} \sin 2 \Lambda \tau+\mathcal{O}\left(1 / S^{3}\right) .
\end{aligned}
$$

Note that the swinging motion is linearly stable for arbitrarily high values of $\Lambda$.

Tumbling: During tumbling motion the inclination angle $\Psi$ grows very fast, to leading order $\dot{\Psi} \simeq-\Lambda$. We assume a slow dynamics of the shape $\beta_{0}(\tau)$, superimposed by small but fast oscillations $\beta_{1}(\Lambda \tau)$. From the leading order equations of 35 , 37) we obtain

$$
\begin{aligned}
& \phi_{1}(\tau)=-\frac{1}{2 \Lambda \sin \beta_{0}} \sin (2 \Lambda \tau), \\
& \beta_{1}(\tau)=\frac{\cos \beta_{0}}{2 \Lambda} \cos 2 \Lambda \tau .
\end{aligned}
$$

The equations at order $1 / \Lambda$ give

$$
\dot{\phi}_{0}=-\frac{1}{S} \frac{\sin 2 \phi_{0}}{\sin \beta_{0}}-\left\langle\frac{\cos 2 \Lambda \tau}{\sin \beta_{0}+\beta_{1}}\right\rangle
$$




$$
\begin{aligned}
& =-\frac{1}{S} \frac{\sin 2 \phi_{0}}{\sin \beta_{0}}-\frac{1}{4 \Lambda} \frac{1+\sin ^{2} \beta_{0}}{\sin ^{2} \beta_{0}} \\
\dot{\beta}_{0} & =-\frac{1}{S} \cot \hat{\beta} \sin \beta_{0}+\frac{1}{S} \cos \beta_{0} \cos 2 \phi_{0}+\frac{1}{S}\langle\cos \beta \sin 2 \Psi\rangle,
\end{aligned}
$$

where $\langle\cdots\rangle$ denotes averaging over one period of the fast oscillations. The average of the cross term $\langle\cos \beta \sin 2 \Psi\rangle$ on the right hand side of 61$\rangle$ is zero, since the oscillations of $\beta$ and $\Psi$ have a relative phase shift of $\pi$.

Stable tumbling regime: Equations 60 61) admit a stationary solution determined by the conditions

$$
\begin{aligned}
\sin 2 \phi_{0} & =-\frac{1+\sin ^{2} \beta_{0}}{\sin \beta_{0}} \frac{S}{4 \Lambda}, \\
\cos 2 \phi_{0} & =\frac{\tan \beta_{0}}{\tan \hat{\beta}} .
\end{aligned}
$$

The solution $\beta(\Lambda / S)$ of this system, which is shown in figure 12 , has two branches. The upper branch was found to be numerically stable. For $\Lambda / S \rightarrow \infty$ the stable branch approaches $\beta_{0} \rightarrow \hat{\beta}$ (with $\phi_{0} \rightarrow 0$ ). On the unstable branch we have $\beta_{0} \rightarrow 0$ (with $\left.\phi_{0} \rightarrow \pi / 4\right)$ as $\Lambda / S \rightarrow \infty$. We can avoid multi-valued functions by instead writing $\Lambda / S$ as a function of $\beta_{0}$

$$
\left.\frac{\Lambda}{S}\right|_{\mathrm{tu}}=\frac{\left(3-\cos 2 \beta_{0}^{\mathrm{tu}}\right) \tan \hat{\beta}}{8 \sin \beta_{0}^{\mathrm{tu}} \sqrt{\tan ^{2} \hat{\beta}-\tan ^{2} \beta_{0}^{\mathrm{tu}}}} .
$$

The tumbling solution only exists above a critical ratio $\Lambda /\left.S\right|_{\text {crit. }} \simeq 0.627$. This finding corroborates the first order phase transition found numerically. An analytical expression for $\Lambda /\left.S\right|_{\text {crit. }}$ can be derived by minimizing the right hand side of (64). However, the resulting expression as a function of $\hat{\beta}$ is too involved to be reproduced here.

The flow field of the shape parameter $\beta$ is shown in figure 12 for $S=10$. Below $\Lambda \simeq 6.27$, there is only one (oscillating) stationary solution for $\beta$, which is the stable transient motion. Above $\Lambda \simeq 6.27$, both the stable and unstable tumbling branch and the swinging solution coexist. If we start with an initial capsule shape $\beta(0)$ above the value $\beta_{0}^{\text {tu }}(\Lambda / S)$ on the unstable branch, the capsule tumbles in the long time limit. Starting with an initial capsule shape $\beta(0)$ below the value $\beta_{0}^{\text {tu }}(\Lambda / S)$ on the unstable branch, the capsule will swing. To check the coexistence of both motions, we solved the dynamics now starting with the initial shape $\beta(0)=\arcsin (1 / \Lambda)$. With this carefully chosen initial condition, transient motion is indeed stable for arbitrarily large values of $\Lambda$.

Initial tumbling in the transient regime: Equations 60 61 can also lead to more insight regarding the transient regime. Since no tumbling solution is stable for $\Lambda / S<0.627$, the shape parameter monotonously decreases until it reaches $\beta_{0}=$ $\arcsin 1 / \Lambda \approx 1 / \Lambda$. While $\beta(t)$ is of the order one, the slow shape dynamics is given by 6061 , which can be easily solved numerically. It is, however, instructive to consider the parameters $1 \ll \Lambda \ll S$ well inside the transient regime away from the phase 
boundaries. Here, the equations of motion simplify to

$$
\begin{aligned}
& \dot{\phi}_{0}^{\operatorname{tr}}=-\frac{1}{4 \Lambda} \frac{1+\sin ^{2} \beta_{0}}{\sin ^{2} \beta_{0}}, \\
& \dot{\beta}_{0}^{\operatorname{tr}}=-\frac{1}{S} \cot \hat{\beta} \sin \beta_{0} .
\end{aligned}
$$

We see that two slow time scales become relevant: The phase angle $\phi$ changes monotonously on a typical time scale $\Lambda$, while the shape relaxes on the much longer

time scale $S$. During this time the capsule tumbles. The decreasing phase angle $\phi$ during the initial tumbling motion is also visible in figure 11.

\section{Perspectives}

In this paper we have summarized the current state of research concerning the dynamics of capsules in hydrodynamic shear flow. We have reviewed and categorized the phenomenology observed in experiments and in numerical simulations. In the review part, we focused on the small deformation of initially spherical capsules in shear flow and on the formation of wrinkles on polymerized membranes. For initially non-spherical capsules, the shape memory effect drastically alters the dynamics, leading to a swinging motion. A reduced model with fixed shape explains the swinging behaviour at low viscosity contrast, but is at variance with numerical results at large shear rate. We have solved this discrepancy by systematically expanding the equations of motion for small deformations. Shape changes were found to be paramount to understanding the dynamics. The disputed intermittent regime was found to be an artefact of artificially fixing the capsule shape. In the relevant parameter range the motion is rather a transient one as suggested by numerical evidence. We have obtained analytical expressions for the phase boundaries at strong flow and have identified the swinging to transient transition as being of second order, the transient to tumbling transition as being of first order. In the tumbling regime a swinging motion is also stable for carefully chosen initial conditions.

Until recently, all theoretical and experimental works have focused on soft objects in time-constant flow. The dynamics within the reduced model with constant shape was completely solved analytically for arbitrary time-dependent flow in the quasi-spherical limit [34. Here, the shear flow oscillates around a finite positive value. With suitable modulation of the shear rate, resonant tumbling motion can be excited, even though the mean shear rate lies inside the swinging regime for constant flow. First experiments in time-varying external flow considered red blood cells [18, where the shear rate oscillates with a vanishing mean value. The focus of this study was on the deformation and relaxation of the shape. The oscillating shape showed a time lag with respect to the shear stress of the flow. The shape changes were characterized by a rapid deformation followed by a slow relaxation. Within the expanded reduced model, Noguchi [78] found a qualitatively similar behaviour, deducing that in the experiment the red blood cells have been in the low-frequency swinging regime. For vanishing mean shear rate, there is 
a rich phase behaviour consisting of swinging and tumbling motions and shape changes. For finite mean shear rates, the shape changes also lag behind the oscillating shear stress. These results imply that shape changes might be important in time-varying flow.

How the motion in time-dependent shear flow is altered by allowing shape changes is an open question worthwhile pursuing. We expect particularly interesting effects in the tumbling regime, where two modes of motion are simultaneously stable with their own basin of attraction. When changing the flow parameters periodically, hysteresis might play an important role.

Shear flow above a substrate to which a capsule adheres could lead to dynamical unbinding, or detachment. For vesicles, this effect has been studied both theoretically [79, 80, 81] and in experiments [82, 83, 84. For capsules, the equilibrium shape of such a bound configuration has been calculated as a function of adhesion energy and material parameters [85, 86]. It would now be interesting to investigate how shear flow affects such shapes and how shear-induced detachment of capsules differs from that of vesicles.

A related, but different open area of research is the influence of thermal fluctuations. While thermal noise is energetically not as relevant for capsules as it is for vesicles, it is known to influence phase transitions. Thermal fluctuations can be added to the equations of motion for capsules as has been done for three-dimensional quasispherical vesicles [87, 88, 89, 61] and two-dimensional quasi-circular vesicles [90, 91]. In Steinberg's group [92], thermally induced fluctuations in shape and inclination angle of a giant vesicle in a stationary tank-treading regime was investigated as a function of the membrane area and viscosity contrast.

Finally, it looks promising to treat the stationary motion of capsules and vesicles as examples of non-equilibrium steady states from the perspective of stochastic thermodynamics [93, 94]. One could then ask for the entropy production in such states and its behaviour at these dynamical transitions. Likewise, the response of swinging and tumbling motion to additional small perturbations could then be expressed by correlation functions in the corresponding steady state [95]. Within such a perspective, vesicles and capsules could become two-dimensional paradigms for exploring these novel concepts which so far have mainly been studied for zero-dimensional and onedimensional objects by using, respectively, colloidal particles and polymers.

\section{Appendix A. Vector spherical harmonics}

A complete orthonormal basis for three-dimensional vector fields on the sphere is given by the vector spherical harmonics [96] which are defined as

$$
\begin{aligned}
\mathbf{Y}_{l}^{m}(\theta, \phi) & \equiv Y_{l}^{m}(\theta, \phi) \mathbf{e}_{r}(\theta, \phi), \\
\mathbf{\Psi}_{l}^{m}(\theta, \phi) & \equiv \frac{1}{\sqrt{l(l+1)}} \hat{\nabla} Y_{l}^{m}(\theta, \phi), \\
\boldsymbol{\Phi}_{l}^{m}(\theta, \phi) & \equiv \mathbf{e}_{r} \times \mathbf{\Psi}_{l}^{m}(\theta, \phi),
\end{aligned}
$$


where $Y_{l}^{m}(\theta, \phi)$ are the standard scalar spherical harmonics $(l=0,1, \ldots$ and $m=$ $-l,-l+1, \ldots, l)$, the operator

$$
\hat{\nabla} \equiv \mathbf{e}_{\theta} \partial_{\theta}+\frac{1}{\sin \theta} \mathbf{e}_{\phi} \partial_{\phi}
$$

is the surface gradient on the sphere, and the scalar product of complex vector fields a and $\mathbf{b}$ is defined as

$$
\langle\mathbf{a}, \mathbf{b}\rangle \equiv \int_{0}^{2 \pi} \int_{0}^{\pi} \mathbf{a}^{*} \cdot \mathbf{b} \sin \theta \mathrm{d} \theta \mathrm{d} \phi
$$

with index $*$ denoting complex conjugate.

\section{Appendix B. Elastic energy}

Each membrane point can be labelled by two Lagrangian coordinates $\left(\theta_{R}, \phi_{R}\right)$, or equivalently by points $\mathbf{x}\left(\theta_{R}, \phi_{R}\right)$ on a hypothetical reference sphere, if $\left(\theta_{R}, \phi_{R}\right)$ are interpreted as spherical coordinates. Here,

$$
\mathbf{x}\left(\theta_{\mathrm{R}}, \phi_{\mathrm{R}}\right) \equiv R\left[\sin \left(\theta_{\mathrm{R}}\right) \cos \left(\phi_{\mathrm{R}}\right), \sin \left(\theta_{\mathrm{R}}\right) \sin \left(\phi_{\mathrm{R}}\right), \cos \left(\theta_{\mathrm{R}}\right)\right]^{T}
$$

are the Cartesian coordinates of a point on the surface of a sphere with radius $R$ with given spherical coordinates. The actual membrane configuration is then constructed in two steps: First, the reference sphere is rotated by a three-dimensional rotation $\mathbf{\Phi} \equiv \Phi \mathbf{n}$ by an angle $\Phi$ around an axis of rotation $\mathbf{n}$. The position of the membrane point is then given by $\mathbf{D}_{\boldsymbol{\Phi}} \mathbf{x}\left(\theta_{\mathrm{R}}, \phi_{\mathrm{R}}\right) \equiv \mathbf{x}(\theta, \phi)$. Here, $\mathbf{D}_{\boldsymbol{\Phi}}$ represents the Cartesian rotation matrix. The analytical expressions for the rotated spherical coordinates $(\theta, \phi)$ as a function of the original coordinates $\left(\theta_{\mathrm{R}}, \phi_{\mathrm{R}}\right)$ and the rotation angle $\boldsymbol{\Phi}$ are involved. Only in the special case where $\mathbf{n}=\mathbf{e}_{z}$ they simplify to $\theta=\theta_{\mathrm{R}}, \phi=\phi_{\mathrm{R}}+\Phi$.

In a second step, the points of the rotated sphere are displaced by a deformation vector $\epsilon R \mathbf{u}(\theta, \phi)$. The final position of a membrane point is

$$
\mathbf{r}\left(\theta_{\mathrm{R}}, \phi_{\mathrm{R}}\right)[\mathbf{\Phi}, \mathbf{u}]=\mathbf{D}_{\boldsymbol{\Phi}} \mathbf{x}\left(\theta_{\mathrm{R}}, \phi_{\mathrm{R}}\right)+\epsilon R \mathbf{u}(\theta, \phi)
$$

We have factored the capsule radius out of the definition of $\mathbf{u}$ to make $\mathbf{u}$ dimensionless. The pre-factor $\epsilon$ formally a small parameter for our perturbation expansion. Its specific value will be chosen below for a convenient normalisation of $\mathbf{u}$. The equilibrium shape of a capsule is given by a specific deformation $\hat{\mathbf{u}}$ (we can set $\hat{\boldsymbol{\Phi}}=\mathbf{0}$ without loss of generality).

The elastic energy of such a deformation is modelled using an Evans-Skalak constitutive law with locally and globally incompressible membrane [1]. In the quasispherical limit the energy can be written as

$$
\mathcal{H}[\mathbf{u}, \boldsymbol{\Phi}] \equiv \mu \int d A \epsilon: \epsilon+\int d A \sigma .
$$

The local tension $\sigma$ is a Lagrange multiplier to ensure local and global area incompressibility. The strain tensor

$$
\epsilon\left(\theta_{\mathrm{R}}, \phi_{\mathrm{R}}\right) \equiv \frac{1}{2}\left[\mathbf{g}[\mathbf{u}](\theta, \phi)-\hat{D}_{\mathbf{\Phi}} \mathbf{g}[\hat{\mathbf{u}}](\theta, \phi)\right]
$$


measures the difference in the metric tensors $\mathbf{g}$ between the equilibrium and the actual configuration. Note that the metrics are evaluated at the same material point $\left(\theta_{R}, \phi_{R}\right)$, which are in general at different Cartesian positions in both configurations. One metric tensor must therefore be moved to the other position. This is performed using the rotation operator $\hat{D}_{\boldsymbol{\Phi}}$, which transforms the tensor components accordingly. We can simplify the calculation of the elastic energy considerably by taking advantage of the fact that the strain tensor is linear in the deformation in the quasi-spherical approximation. The strain tensor and the corresponding elastic energy $\mathcal{H}_{0}[\mathbf{u}]$ for a deformation from a spherical equilibrium shape ( $\hat{\mathbf{u}} \equiv 0)$ without area constraint has already be calculated in the literature [16, 15]. The corresponding expression for a deformation from a nonspherical equilibrium shape is then simply $\mathcal{H}_{0}\left[\mathbf{u}-\hat{D}_{\boldsymbol{\Phi}} \hat{\mathbf{u}}\right]$.

To take advantage of the spherical symmetry of the reference sphere, we switch to a vector spherical harmonics representation of the deformation field

$$
\mathbf{u} \equiv \frac{u_{00}^{Y}}{\sqrt{4 \pi}} \mathbf{e}_{r}+\sum_{l m}\left[u_{l m}^{Y} \mathbf{Y}_{l}^{m}+u_{l m}^{\Psi} \mathbf{\Psi}_{l}^{m}+u_{l m}^{\Phi} \boldsymbol{\Phi}_{l}^{m}\right]
$$

Here and in the following, the sum over $l$ and $m$ runs from $l \geq 1$ with $-l \leq m \leq l$. The vector spherical harmonics are defined in Appendix A. Expressions for the volume, area, and bending energy in terms of the deformation amplitudes $u_{l m}^{Y}$ are derived in [89], while expressions for the elastic energy against shearing and stretching in the case of spherical capsules can be found in [15].

To obey volume conservation, the constant $u_{00}$ is a function of the higher mode amplitudes and can be eliminated. The excess area is then given by

$$
\Delta=\epsilon^{2} \sum_{l m}\left|u_{l m}^{Y}\right|^{2} \frac{(l+2)(l-1)}{2} \equiv \frac{\epsilon^{2}}{2} \sum_{l m} F_{l}\left|u_{l m}^{Y}\right|^{2} .
$$

The shear modes $u_{l m}^{\Phi}$ completely decouple from the other two deformation modes and are omitted in the following. Taking advantage of the local area constraint $2\left(u_{l m}^{Y}-\hat{D}_{\boldsymbol{\Phi}} \hat{u}_{l m}^{Y}\right)=\sqrt{l(l+1)} u_{l m}^{\Psi}$ we can write the elastic energy as

$$
\begin{aligned}
\mathcal{H}[\mathbf{u}, \boldsymbol{\Phi}]= & \epsilon^{2} R^{2} \frac{\mu}{2} \sum_{l m} \frac{4\left(l^{2}+l-2\right)}{l(l+1)}\left|u_{l m}^{Y}-\hat{D}_{\boldsymbol{\Phi}} \hat{u}_{l m}^{Y}\right|^{2} \\
& +\epsilon^{2} R^{2} \frac{\bar{\sigma}}{2} \sum_{l m} F_{l}\left|u_{l m}^{Y}\right|^{2} .
\end{aligned}
$$

Here, $\bar{\sigma}$ denotes the homogeneous tension $\bar{\sigma} \equiv \int d A \sigma / A$ ensuring global area compressibility.

Although $\epsilon$ is formally a free parameter signifying the order of magnitude of the terms, we chose to set

$$
\epsilon \equiv \sqrt{\Delta / 2}
$$

With this choice the normalization of the $\mathbf{u}_{l m}$ reads

$$
\frac{1}{4} \sum_{l m} F_{l}\left|u_{l m}^{Y}\right|^{2}=1
$$


When only $l=2$ modes are excited, this simplifies to $\sum_{m}\left|u_{2 m}^{Y}\right|^{2}=1$.

In the spherical harmonic representation the rotation operator reduces to the Wigner rotation matrices

$$
\hat{D}_{\mathbf{\Phi}} u_{l m}^{s}=\sum_{m^{\prime}} D_{m^{\prime}, m}^{l}(\mathbf{\Phi}) u_{l m^{\prime}}^{s}
$$

The elastic force in radial direction is given by

$$
\begin{aligned}
f_{l m}^{Y \mathrm{el}} & \equiv-\frac{\partial H[\mathbf{u}]}{\epsilon R^{3} \partial u_{l m}^{Y *}} \\
& =-\frac{\mu \epsilon}{R} \frac{4\left(l^{2}+l-2\right)}{l(l+1)}\left[u_{l m}^{Y}-\sum D_{m m^{\prime}}^{l}(\boldsymbol{\Phi}) \hat{u}_{l m^{\prime}}\right]-\frac{\epsilon \bar{\sigma}}{R} F_{l} u_{l m}^{Y} .
\end{aligned}
$$

where the global area constraint contributes a tensile force. Additional forces ensure local area incompressibility under the capsule motion.

\section{Appendix C. Equations of motion}

Since Stokes' equations are linear, any solution can be written as the superposition of different flow fields. Moreover, we can chose the boundary conditions at the membrane and at infinity of each particular solution to our advantage, as long as the overall solution matches the correct boundary conditions given by the force balance and the external flow. We can thus separate the flow fields induced by an elongational and rotational part and flow induced by the membrane forces and torques

$$
\mathbf{v}(\mathbf{r})=\mathbf{v}_{\text {ind }}(\mathbf{r})+\mathbf{v}_{s}(\mathbf{r})+\mathbf{v}_{\omega}(\mathbf{r}) .
$$

For the rotational velocity field $\mathbf{v}_{\omega}$ we take the corresponding component of the external flow throughout

$$
\mathbf{v}_{\omega}=\omega \mathbf{e}_{z} \times \mathbf{r} .
$$

Since the hydrodynamic stress of a purely rotating fluid vanishes, this flow does not induce forces at the membrane. As the elastic energy of the membrane depends on $\boldsymbol{\Phi}$, a torque is exerted on the surrounding fluid. This torque is, however, of the order $\epsilon^{2}$ and is neglected in the following. The no-slip boundary condition at the capsule membrane therefore requires

$$
\dot{\Phi}=\omega \mathbf{e}_{z} .
$$

For $\mathbf{v}_{s}$ we chose a flow which matches the elongational part of the external flow at infinity and vanishes at the sphere. While such a flow field produces surface forces on the capsule membrane, these forces only depend on the outer viscosity $\eta_{o}$. The resulting force at $r=R$ is

$$
\mathbf{f}_{2, m}^{s}=5 i s \eta_{o}\left(\delta_{m,-2}-\delta_{m, 2}\right) \sqrt{\frac{2 \pi}{15}}\left(2 \mathbf{Y}_{2}^{m}+\sqrt{6} \mathbf{\Psi}_{2}^{m}\right) .
$$

The remaining flow field is induced by the surface forces of the capsule membrane and of the hydrodynamic stress jump of the extensional flow. The induced flow field 
itself vanishes away from the capsule. In other words, the induced flow field is the same as in an ambient fluid. The global flow field can be found explicitly (see [89]), but is of no interest here. We only need to know the value of the flow field at the capsule. The linear relationship between the surface forces and the induced velocity is given by the (inverse) Oseen tensor

$$
\mathcal{O}_{l}^{-1} \cdot \mathbf{v}_{l, m}^{\text {ind }}=\mathbf{f}_{l, m}^{s}+\mathbf{f}_{l, m}^{\mathrm{el}}+\mathbf{f}_{l, m}^{\sigma} .
$$

The viscosity contrast only appears in the expression of the Oseen tensor, which reads explicitly [15]

$$
\mathcal{O}_{l}^{-1} \equiv \frac{1}{R}\left(\begin{array}{cc}
\eta_{i} \frac{2 l^{2}+l+3}{l}+\eta_{o} \frac{2 l^{2}+3 l+4}{l+1} & -3 \eta_{i} \sqrt{\frac{l+1}{l}}-3 \eta_{o} \sqrt{\frac{l}{l+1}} \\
-3 \eta_{i} \sqrt{\frac{l+1}{l}}-3 \eta_{o} \sqrt{\frac{l}{l+1}} & \eta_{i}(2 l+1)+\eta_{o}(2 l+1)
\end{array}\right) .
$$

Again, local area incompressibility enforces a coupling between radial and tangential flow.

Finally, the membrane is advected with the resulting flow owing to the no-slip boundary condition.

The membrane dynamics is thus governed by the equation

$$
D_{t} \mathbf{u}_{l m}=\mathbf{v}_{l m}^{s}+\mathcal{O}_{l}\left(\mathbf{f}_{l, m}^{s}+\mathbf{f}_{l, m}^{\mathrm{el}}+\mathbf{f}_{l, m}^{\sigma}\right) \text {. }
$$

Here, the material time derivative of the deformation amplitude reads

$$
D_{t} \mathbf{u} \equiv\left(\partial_{t}+(\mathbf{v} \cdot \nabla)\right) \mathbf{u}
$$

In the following we only take the rotational part of the flow when calculating the advection, since the other contributions are smaller by a factor $\epsilon$. In other words, we use

$$
D_{t} \mathbf{u}=\left(\partial_{t}+(\dot{\boldsymbol{\Phi}} \times \mathbf{r}) \cdot \nabla\right) \mathbf{u}=\left(\partial_{t}+\omega \partial_{\phi}\right) \mathbf{u} .
$$

This is justified because the rotational velocity is an order of magnitude larger than the velocity components leading to a membrane deformation. Equations (B.11, C.3, C.4. C.5. C.7 C.9 specify the dynamics of the capsule in the quasi-spherical limit completely.

We see that in linear external flow only the $l=2$ modes can be excited. We therefore restrict ourselves to only taking $l=2$ deformation modes. Since the radial and tangential modes are coupled by the local area constraint, we can eliminate the latter modes. By redefining the time scale $\tau$ and introducing dimensionless strengths of the rotational $\Lambda$ and elongational $S$ flow analogously to [97] (see (38) ) the equations of motions simplify to (omitting the now redundant $Y$ and $l=2$ indices)

$$
\begin{aligned}
\partial_{\tau} \phi \quad & \Lambda \\
\left(\partial_{\tau}-i \Lambda m\right) u_{m}= & \sum_{m^{\prime}}\left(\delta_{m m^{\prime}}-u_{m} u_{m^{\prime}}^{*}\right) \times \\
& {\left[S^{-1} \exp \left(-i m^{\prime} \Phi\right) \hat{u}_{m^{\prime}} / \sin \hat{\beta}-i\left(\delta_{m^{\prime}, 2}-\delta_{m^{\prime},-2}\right)\right] . }
\end{aligned}
$$

These equations are formally very similar to the equations of motions for vesicles in (48) of [97]. Let us first search for solutions which show the same mirror symmetry with 
respect to the $x y$-plane as the external flow. Thus, only the modes with $m=-2,0,2$ are non-zero (also for $\hat{u}_{m}$ ). Since the modes are normalized to $\sum_{m}\left|u_{m}\right|^{2}=1$, we can employ the parametrisation

$$
\begin{aligned}
& u_{0} \equiv \cos (\beta) \\
& u_{2} \equiv \sin (\beta) \exp (-2 i \Psi) / \sqrt{2} \\
& u_{-2} \equiv \sin (\beta) \exp (2 i \Psi) / \sqrt{2}
\end{aligned}
$$

Here, $\Psi \in[-\pi / 2, \pi / 2]$ is the inclination angle of the capsule with respect to the $x$-axis, $\beta \in[0, \pi / 2]$. To compare with the equations of motion 21,22$)$ in the reduced Skotheim model, (cf. section 5) it is advantageous to switch to the phase angle $\phi$ in the capsule frame rather than the phase angle $\Phi$ in the laboratory frame. Both angles are related by the inclination angle, $\Phi \equiv \phi+\Psi$. We thus arrive at the equations of motion (35) 37).

\section{References}

[1] C. Pozrikidis, editor. Modelling and simulation of capsules and biolocical cells. Chapman \& Hall/CRC, 2003.

[2] H. Möhwald, E. Donath, and G. Sukhorukov. Smart capsules. New York: Wiley VCH, 2003.

[3] A. Fery and R. Weinkamer. Mechanical properties of micro- and nanocapsules: Single-capsule measurements. Polymer, 48:7221, 2007.

[4] M. Abkarian and A. Viallat. Vesicles and red blood cells in shear flow. Soft matter, 4:653, 2008.

[5] D. Barthès-Biesel. Capsule motion in flow: Deformation and membrane buckling. C. R. Physique, 10:764, 2009.

[6] U. Seifert. Configurations of fluid membranes and vesicles. Adv. Physics, 46:13, 1997.

[7] H. Rehage, M. Husmann, and A. Walter. From two-dimensional model networks to microcapsules. Rheol. Acta, 41:292, 2002.

[8] D. E. Discher and P. Carl. New insights into red cell network structure, elasticity, and spectrin unfolding - a current review. Cell. Mol. Biol. Lett., 6:593, 2001.

[9] S. A. Patwardhan and K. G. Das. Microencapsulation. In K. G. Das, editor, Controlled release technology - bioengineering aspects, pages 121-141. Wiley, New York, 1983.

[10] A. D. Dinsmore, M. F. Hsu, M. G. Nikolaides, M. Marquez, A. R. Bausch, and D.A. Weitz. Colloidosomes: Selectively permeable capsules composed of colloidal particles. Science, 298:1006, 2002.

[11] S. R. Keller and R. Skalak. Motion of a tank-treading ellipsoidal particle in a shear-flow. J. Fluid. Mech., 120:27, 1982.

[12] T. M. Fischer. Shape memory of human red blood cells. Biophys. J., 86:3304, 2004.

[13] J. M. Skotheim and T. W. Secomb. Red blood cells and other nonspherical capsules in shear flow: Oscillatory dynamics and the tank-treading-to-tumbling transition. Phys. Rev. Lett., 98:078301, 2007.

[14] M. Abkarian, M. Faivre, and A. Viallat. Swinging of red blood cells under shear flow. Phys. Rev. Lett., 98:188302, 2007.

[15] S. B. Rochal, V. L. Lorman, and G. Mennessier. Viscoelastic dynamics of spherical composite vesicles. Phys. Rev. E, 71, 2005.

[16] D. Barthès-Biesel. Motion of a spherical microcapsule freely suspended in a linear shear flow. J. Fluid. Mech., 100:831, 1980.

[17] D. Barthès-Biesel and J. M. Rallison. The time-dependent deformation of a capsule freely suspended in a linear shear flow. J. Fluid. Mech., 113:251, 1981.

[18] N. Watanabe, H. Kataoka, T. Yasuda, and S. Takatani. Dynamic deformation and recovery 
response of red blood cells to a cyclically reversing shear flow: Effects of frequency of cyclically reversing shear flow and shear stress level. Biophys. J., 91:1984, 2006.

[19] T. M. Fischer. Tank-tread frequency of the red cell membrane: Dependence on the viscosity of the suspending medium. Biophys. J., 93:2553, 2007.

[20] C. Misbah. Vacillating breathing and tumbling of vesicles under shear flow. Phys. Rev. Lett., 96:28104, 2006.

[21] H. Noguchi and G. Gompper. Swinging and tumbling of fluid vesicles in shear flow. Phys. Rev. Lett., 98:128103, 2007.

[22] V. V. Lebedev, K. S. Turitsyn, and S. S. Vergeles. Dynamics of nearly spherical vesicles in an external flow. Phys. Rev. Lett., 99:218101, 2007.

[23] X. Z. Li, D. Barthès-Biesel, and A. Helmy. Large deformations and burst of a capsule freely suspended in an elongational flow. J. Fluid. Mech., 187:179, 1988.

[24] A. Leyrat-Maurin, A. Drochon, and D. Barthes-Biesel. Flow of a capsule through a constriction - application to cell filtration. J. Phys. Paris III, 3:1051, 1993.

[25] A. Leyrat-Maurin and D. Barthes-Biesel. Motion of a deformable capsule through a hyperbolic constriction. J. Fluid. Mech., 279:135, 1994.

[26] H. Zhou and C. Pozrikidis. Deformation of liquid capsules with incompressible interfaces in simple shear flow. J. Fluid. Mech., 283:175, 1995.

[27] C. Pozrikidis. Finite deformation of liquid capsules enclosed by elastic membranes in simple shearflow. J. Fluid. Mech., 297:123, 1995.

[28] S. Ramanujan and C. Pozrikidis. Deformation of liquid capsules enclosed by elastic membranes in simple shear flow: Large deformations and the effect of fluid viscosities. J. Fluid Mech., 361:117, 1998.

[29] C. Pozrikidis. Effect of bending stiffness on the deformation of liquid capsules enclosed by elastic membranes in simple shear flow. J. Fluid. Mech., 440:269, 2001.

[30] C. Pozrikidis. Numerical simulation of the flow-induced deformation of red blood cells. Ann. Biomed. Eng., 31:1194, 2003.

[31] E. Lac. Déformation et convection d'une capsule dans un écoulement de Stokes tridimenionnel infini. PhD thesis, Université Compiegne, 2003.

[32] E. Lac, D. Barthès-Biesel, N. A. Pelekasis, and J. Tsamopoulos. Spherical capsules in threedimensional unbounded Stokes flows: effect of the membrane constitutive law and onset of buckling. J. Fluid. Mech., 516:303, 2004.

[33] E. Lac and D. Barthès-Biesel. Deformation of a capsule in shear flow: Effect of membrane prestress. Phys. Fluids, 17:72105, 2005.

[34] S. Kessler, R. Finken, and U. Seifert. Swinging and tumbling of elastic capsules in shear flow. J. Fluid. Mech., 605:207, 2007.

[35] C. Pozrikidis. A spectral collocation method with triangular boundary elements. Eng. Anal. Bound. Elem., 30:315, 2006.

[36] Y. C. Wang and P. Dimitrkopoulos. A three-dimensional spectral boundary element algorithm for interfacial dynamics in Stokes flow. Phys. Fluids, 18:82106, 2006.

[37] P. Dimitrakopoulos. Interfacial dynamics in Stokes flow via a three-dimensional fully-implicit interfacial spectral boundary element algorithm. J. Comput. Phys., 225:408, 2007.

[38] W. R. Dodson III and P. Dimitrakopoulos. Spindles, cusps, and bifurcation for capsules in Stokes flow. Phys. Rev. Lett., 101:208102, 2008.

[39] C. S. Peskin. Numerical-analysis of blood-flow in heart. J. Comput. Phys., 25:220, 1977.

[40] C. S. Peskin. The immersed boundary method. Acta Numerica, page 479, 2002.

[41] C. D. Eggleton and A. S. Popel. Large deformation of red blood cell ghosts in a simple shear flow. Phys. Fluids, 10:1834, 1998.

[42] P. Bagchi and R. M. Kalluri. Dynamics of nonspherical capsules in shear flow. Phys. Rev. E, 80:016307, 2009.

[43] Y. Sui, Y. T. Chew, P. Roy, X. B. Chen, and H. T. Low. Transient deformation of elastic capsules 
in shear flow: Effect of membrane bending stiffness. Phys. Rev. E, 75:066301, 2007.

[44] Y. Sui, Y. T. Chew, and H. T. Low. A lattice Boltzmann study on the large deformation of red blood cells in shear flow. Int. J. Mod. Phys. C, 18:993, 2007.

[45] Y. Sui, Y. T. Chew, P. Roy, and H. T. Low. Effect of membrane bending stiffness on the deformation of elastic capsules in extensional flow: A lattice Boltzmann study. Int. J. Mod. Phys. C, 18:1277, 2007.

[46] Y. Sui, H. T. Low, Y. T. Chew, and P. Roy. Tank-treading, swinging, and tumbling of liquid-filled elastic capsules in shear flow. Phys. Rev. E, 77:016310, 2008.

[47] Y. Sui, Y. T. Chew, P. Roy, Y. P. Cheng, and H. T. Low. Dynamic motion of red blood cells in simple shear flow. Phys. Fluids, 20:112106, 2008.

[48] Y. Sui, X. B. Chen, Y. T. Chew, P. Roy, and H. T. Low. Numerical simulation of capsule deformation in simple shear flow. Comput. Fluids, 39:242, 2010.

[49] Y. Sui, H.T. Low, Y. T. Chew, and P. Roy. A front-tracking lattice boltzmann method to study flow-induced deformation of three-dimensional capsules. Computers \& Fluids, 39:499, 2010.

[50] S. U. Unverdi and G. Tryggvason. A front-tracking method for viscous, incompressible, multi-fluid flows. J. Comput. Phys., 100:25, 1992.

[51] G. Ma, J. S. Hua, and H. Li. Numerical modeling of the behavior of an elastic capsule in a microchannel flow: The initial motion. Phys. Rev. E, 79:046710, 2009.

[52] Y. Sui, Y. T. Chew, P. Roy, X. B. Chen, and H. T. Low. Transient deformation of elastic capsules in shear flow: Effect of membrane bending stiffness. Phys. Rev. E, 75:66301, 2007.

[53] B. Kaoui, G. H. Ristow, I. Cantat, C. Misbah, and W. Zimmermann. Lateral migration of a two-dimensional vesicle in unbounded poiseuille flow. Phys. Rev. E, 77:021903, 2008.

[54] M. Kraus, W. Wintz, U. Seifert, and R. Lipowsky. Fluid vesicles in shear flow. Phys. Rev. Lett., $77: 3685,1996$.

[55] T. Biben, A. Farutin, and C. Misbah. Numerical study of 3d vesicles under flow: discovery of new peculiar behaviors. arXiv:0912.4702 [cond-mat.soft], 2009.

[56] T. Biben and C. Misbah. Tumbling of vesicles under shear flow within an advected-field approach. Phys. Rev. E, 67:031908, 2003.

[57] J. Beaucourt, F. Rioual, T. Seon, T. Biben, and C. Misbah. Steady to unsteady dynamics of a vesicle in a flow. Phys. Rev. E, 69:011906, 2004.

[58] T. Biben, K. Kassner, and C. Misbah. Phase-field approach to three-dimensional vesicle dynamics. Phys. Rev. E, 72:041921, 2005.

[59] H. Noguchi and G. Gompper. Fluid vesicles with viscous membranes in shear flow. Phys. Rev. Lett., 93:258102, 2004.

[60] H. Noguchi and G. Gompper. Shape transitions of fluid vesicles and red blood cells in capillary flows. Proc. Natl. Acad. Sci. USA, 102:14159, 2005.

[61] H. Noguchi and G. Gompper. Dynamics of fluid vesicles in shear flow: Effect of membrane viscosity and thermal fluctuations. Phys. Rev. E, 72:011901, 2005.

[62] J. L. McWhirter, H. Noguchi, and G. Gompper. Flow-induced clustering and alignment of vesicles and red blood cells in microcapillaries. Proc. Natl. Acad. Sci. USA, 106:6039, 2009.

[63] K. S. Chang and W. L. Olbricht. Experimental studies of the deformation of a synthetic capsule in extensional flow. J. Fluid. Mech., 250:587, 1993.

[64] K. S. Chang and W. L. Olbricht. Experimental studies of the deformation and breakup of a synthetic capsule in steady and unsteady simple shear-flow. J. Fluid. Mech., 250:609, 1993.

[65] A. Walter, H. Rehage, and H. Leonhard. Shear-induced deformations of polyamid microcapsules. Colloid. Polym. Sci., 278:169, 2000.

[66] A. Walter, H. Rehage, and H. Leonhard. Shear induced deformation of microcapsules: shape oscillations and membrane folding. Coll. Surf. A, 183-185:123, 2001.

[67] H. L. Goldsmith and J Marlow. Flow behavior of erythrocytes. 1. rotation and deformation in dilute suspensions. Proc. R. Soc. London, Ser. B, 182:351, 1972.

[68] T. Fischer and H. Schmid-Schönbein. Tank tread motion of red-cell membranes in viscometric 
flow - behavior of intracellular and extracellular markers (with film). Blood Cells, 3:351, 1977.

[69] T. Fischer, M. Stöhrliesen, and H. Schmid-Schönbein. Red-cell as a fluid droplet - tank tread-like motion of human erythrocyte-membrane in shear-flow. Science, 202:894, 1978.

[70] R. Tran-Son-Tay, S. P. Sutera, and P. R. Rao. Determination of red-blood-cell membrane viscosity from rheoscopic observations of tank-treading motion. Biophys. J., 46:65, 1984.

[71] E. Cerda and L. Mahadevan. Geometry and physics of wrinkling. Phys. Rev. Lett., 90:074302, 2003.

[72] R. Finken and U. Seifert. Wrinkling of microcapsules in shear flow. J. Phys.: Condens. Matter, 18:L185, 2006.

[73] S. Kessler, R. Finken, and U. Seifert. Elastic capsules in shear flow: Analytical solutions for constant and time-dependent shear rates. Eur. Phys. J. E, 29:399, 2009.

[74] H. Noguchi. Swinging and synchronized rotations of red blood cells in simple shear flow. Phys. Rev. E, 80:021902, 2009.

[75] E. J. Hinch. Perturbation Methods, chapter 7. Cambridge University Press, 1991.

[76] H. Noguchi. Dynamic modes of microcapsules in steady shear flow: Effects of bending and shear elasticities. arXiv:1003.3152v1 [cond-mat.soft], 2010.

[77] J. Deschamps, V. Kantsler, E. Segre, and V. Steinberg. Dynamics of a vesicle in general flow. Proc. Natl. Acad. Sci. USA, 106:11444, 2009.

[78] H. Noguchi. Dynamic modes of red blood cells in steady and oscillatory shear flows. arXiv:0903.0038v1 [cond-mat.soft], 2009.

[79] I. Cantat and C. Misbah. Lift force and dynamical unbinding of adhering vesicles under shear flow. Phys. Rev. Lett., 83:880, 1999.

[80] U. Seifert. Hydrodynamic lift on bound vesicles. Phys. Rev. Lett., 83:876, 1999.

[81] S. Sukumaran and U. Seifert. Influence of shear flow on vesicles near a wall: A numerical study. Phy. Rev. E, 64:11916, 2001.

[82] B. Lorz, R. Simson, J. Nardi, and E. Sackmann. Weakly adhering vesicles in shear flow: Tanktreading and anomalous lift force. Europhys. Lett., 51:468, 2000.

[83] M. Abkarian and A. Viallat. Dynamics of vesicles in a wall-bounded shear flow. Biophys. J., $89: 1055,2005$.

[84] S. Chatkaew, M. Georgelin, M. Jaeger, and M. Leonetti. Dynamics of vesicle unbinding under axisymmetric flow. Phys. Rev. Lett., 103:248103, 2009.

[85] P. Graf, R. Finken, and U. Seifert. Adhesion of microcapsules. Langmuir, 22:7117, 2006.

[86] R. M. Springman and J. L. Bassani. Snap transitions in adhesion. J. Mech. Phys. Solids, 56:2358, 2008.

[87] E. I. Kats, V. V. Lebedev, and A. R. Muratov. Nearly spherical vesicles: Shape fluctuations. JETP Lett., 63:216, 1996.

[88] K. H. de Haas, C. Blom, D. van den Ende, M. H. G. Duits, and J. Mellema. Deformation of giant lipid bilayer vesicles in shear flow. Phys. Rev. E, 56:7132, 1997.

[89] U. Seifert. Fluid dynamics in hydrodynamic force fields: Formalism and an application to fluctuating quasispherical vesicles in shear flow. Eur. Phys. J. B, 8:405, 1999.

[90] R. Finken, A. Lamura, U. Seifert, and G. Gompper. Two-dimensional fluctuating vesicles in linear shear flow. Eur. Phys. J. E, 25:309, 2008.

[91] S. Messlinger, B. Schmidt, H. Noguchi, and G. Gompper. Dynamical regimes and hydrodynamic lift of viscous vesicles under shear. Phys. Rev. E, 80:011901, 2009.

[92] V. Kantsler and V. Steinberg. Orientation and dynamics of a vesicle in tank-treading motion in shear flow. Phys. Rev. Lett., 95:258101, 2005.

[93] U. Seifert. Stochastic thermodynamics: principles and perspectives. Eur. Phys. J. B, 64:423, 2008.

[94] T. Speck, J. Mehl, and U. Seifert. Role of external flow and frame invariance in stochastic thermodynamics. Phys. Rev. Lett., 100:178302, 2008.

[95] U. Seifert and T. Speck. Fluctuation-dissipation theorem in nonequilibrium steady states. 
Europhys. Lett, 89:10007, 2010.

[96] P. M. Morse and H. Feshbach. Methods of Theoretical Physics, volume Part II. McGraw-Hill, 1953. p. 1298.

[97] V. Lebedev, K. Turitsyn, and S. Vergeles. Nearly spherical vesicles in an external flow. New Journal of Physics, 10:043044, 2008. 